\title{
Infrared thermography for convective heat transfer measurements
}

\author{
Giovanni Maria Carlomagno • Gennaro Cardone
}

Received: 30 July 2009/Revised: 13 January 2010/Accepted: 11 June 2010/Published online: 3 August 2010

(C) The Author(s) 2010. This article is published with open access at Springerlink.com

\begin{abstract}
This paper deals with the evolution of infrared (IR) thermography into a powerful optical tool that can be used in complex fluid flows to either evaluate wall convective heat fluxes or investigate the surface flow field behavior. Measurement of convective heat fluxes must be performed by means of a thermal sensor, where temperatures have to be measured with proper transducers. By correctly choosing the thermal sensor, IR thermography can be successfully exploited to resolve convective heat flux distributions with both steady and transient techniques. When comparing it to standard transducers, the IR camera appears very valuable because it is non-intrusive, it has a high sensitivity (down to $20 \mathrm{mK}$ ), it has a low response time (down to $20 \mu \mathrm{s}$ ), it is fully two dimensional (from $80 \mathrm{k}$ up to $1 \mathrm{M}$ pixels, at $50 \mathrm{~Hz}$ ) and, therefore, it allows for better evaluation of errors due to tangential conduction within the sensor. This paper analyses the capability of IR thermography to perform convective heat transfer measurements and surface visualizations in complex fluid flows. In particular, it includes the following: the necessary radiation theory background, a review of the main IR camera features, a description of the pertinent heat flux sensors, an analysis of the IR image processing methods and a report on some applications to complex fluid flows, ranging from natural convection to hypersonic regime.
\end{abstract}

This review article was a solicited contribution for the issue of Experiments in Fluids dedicated to the contributions of Prof. Donald Rockwell (Vol. 49, 1).

G. M. Carlomagno $(\bowtie) \cdot$ G. Cardone Department of Aerospace Engineering, University of Naples Federico II, Ple Tecchio 80, 80125 Naples, Italy

e-mail: carmagno@unina.it

\section{Introduction}

Measuring heat fluxes in thermo-fluid-dynamics requires both a thermal sensor (with its related thermo-physical model) and temperature transducers. In standard techniques, where temperature is measured by thermocouples, resistance temperature detectors (RTDs) etc., each transducer yields either the heat flux at a single point or the space-averaged one; hence, in terms of spatial resolution, the sensor itself has to be considered as zero-dimensional. This limitation makes measurements particularly troublesome whenever the temperature, and/or heat flux, fields exhibit high spatial variations. Instead, the infrared (IR) camera constitutes a truly two-dimensional transducer, allowing for accurate measurements of surface temperature maps even in the presence of relatively high spatial gradients. Accordingly, the heat flux sensor also becomes two dimensional, as long as the necessary corrections are applied.

When compared to standard techniques, the use of infrared camera as a temperature transducer in convective heat transfer measurement appears advantageous from several points of view. In fact, since IR camera is fully two dimensional (up to $1 \mathrm{M}$ pixels), besides producing a whole temperature field, it permits an easier evaluation of errors due to radiation and tangential conduction; further, it is non-intrusive (e.g., allowing conduction removal through thermocouple or RTD wires), it has high sensitivity (down to $20 \mathrm{mK}$ ) and low response time (down to $20 \mu \mathrm{s}$ ). As such, IR thermography can be effectively employed to measure convective heat fluxes with both steady and transient techniques.

The first IR cameras were developed in the 1960s as offshoots of military programmes but without accuracy features, not mandatory to existing needs. The 1970s 
energy crisis brought government support in Sweden and so, the first radiometric thermal imagers were developed by AGA and Bofors, both Swedish companies. These cameras used a single detector, the image two-dimensionality being achieved by rotating, or oscillating, mirrors and/or refractive elements (such as prisms) which scanned the field of view (FOV) in both vertical and horizontal directions; in fact, they were also called infrared scanning radiometers.

The detector of these radiometers was the photon one, where the release of electrons is directly associated with photon absorption, its main features being a quite short response time and limited spectral response. Such detector requires cooling, well below ambient temperature to allow for rapid scanning, high sensitivity and low noise. In fact, the sensor was often located in the wall of a Dewar vessel filled with liquid nitrogen $(77 \mathrm{~K})$. Subsequent scanning radiometers used various types of cooled photon detectors with lower time constants allowing frame rates of 15-30 Hz. All real-time commercial cameras used cooled photon single detectors with optomechanical scanning well into the 1980s, when (staring) infrared Focal Plane Array (FPA) detectors, having quite adequate time constants to achieve $30-60 \mathrm{~Hz}$ frame rates, were introduced. By using these staring arrays, the infrared camera, long restricted to a point sensing instrument, became an effective twodimensional transducer. IR cameras based on non-cooled FPA thermal detectors (such as microbolometers) emerged in the mid 1990s and led to the development and diffusion of thermal imagers requiring no cooling.

The earliest attempts to measure heat transfer coefficients arose in the hypersonic regime and were performed by using scanners operating in the middle IR band (3-6 $\mu \mathrm{m})$ of the infrared spectrum, at the time called short wave IR band. In particular, the AGA Thermovision 680SWB camera was employed by Thomann and Frisk (1968) to measure the heat flux distribution on the surface of an elastomeric paraboloid in a hypersonic wind tunnel at Mach number $M=7$. The thin-film sensor (see Sect. 4) was used to determine convective heat transfer coefficients, which showed good agreement with data already obtained with different techniques and was encouraging in view of using IR systems for heat flux measurements. Once the method was shown to work, efforts were mainly oriented toward comprehension of potential errors sources which could affect measurement accuracy and especially to developing devices which could ease IR camera use. Compton (1972), at NASA Ames, realized that the bottleneck of IR thermography was data acquisition, storage and processing. The solution was devised in the automation of data processing and this concept finally brought to the systems currently in use.

Apart from heat flux evaluation, the characterization of flow field behavior, with location of boundary layer transition to turbulence and of flow separation and reattachment zones, constituted a subject of great interest to aerodynamicists and efforts were devoted to acquire information on IR camera capability to deal with these phenomena. The boundary layer transition over a flat plate was examined by Peake et al. (1977), who detected the different adiabatic wall temperatures that occur among laminar and turbulent flows.

A first analysis of heat transfer measurements by IR thermography and a review of some of their applications were presented by Carlomagno and de Luca (1989). Gartenberg and Roberts (1992) reported an extensive retrospective on aerodynamic research with infrared cameras.

In the next section, the basic principles of radiation necessary to perform IR thermography are briefly recalled from the user point of view. The following two sections will revise, first, the infrared camera with its main features and, then, the most commonly used heat flux sensors. Thermal restoration of IR images and related processing methods are discussed in Sect. 5. In the last section, a recapitulation table of the main contributions that have appeared in the last two decades is offered and some significant papers are presented and reviewed.

\section{Basic principles of radiation heat transfer}

Heat transfer by radiation is an energy transport mechanism that occurs in the form of electromagnetic waves. Via this heat transfer mode, energy can also travel in vacuum and may be partially absorbed and reflected by a body, or even pass through it. By denoting with $\alpha_{r}$ the radiation fraction being absorbed by the body, with $\rho_{r}$ the fraction being reflected by it and with $\tau_{r}$ the fraction being transmitted (which passes through), energy conservation requires:

$\alpha_{\mathrm{r}}+\rho_{\mathrm{r}}+\tau_{\mathrm{r}}=1$

where $\alpha_{r}, \rho_{r}$ and $\tau_{r}$ are, respectively called absorptivity, reflectivity and transmissivity coefficients of the body under consideration. As shown later, these coefficients may depend on both radiation wavelength (spectral) and propagation direction (directional).

Radiation is emitted by all bodies at an absolute temperature $T>0$ and, for non-transparent bodies $\left(\tau_{r}=0\right)$, it origins from their surface only. The body which emits the greatest amount of energy at a given temperature is called black body.

The law that prescribes the energy flux (energy rate per unit body area) per wavelength (spectral hemispherical emissive power) $I^{b}(\lambda)\left[\mathrm{W} / \mathrm{m}^{3}\right]$, which is emitted by a black body in the hemisphere outside its surface, is the Planck's law of radiation: 
$I^{b}(\lambda)=\frac{C_{1}}{\lambda^{5}\left(e^{C_{2} / \lambda T}-1\right)}$

where $\lambda$ is the radiation wavelength $(m), T$ the absolute black body temperature $(K)$ and $C_{1}$ and $C_{2}$ the first and the second universal radiation constants, respectively equal to $3.7418 \times 10^{-16} \mathrm{Wm}^{2}$ and $1.4388 \times 10^{-2} \mathrm{mK}$. Equation (2) shows that $I^{b}$ goes to zero for both $\lambda \rightarrow 0$ and $\lambda \rightarrow \infty$. It has to be noted that, for a black body, the radiation intensity (per unit solid angle) based on the body area projected normally to the direction of emission is independent of the latter.

The electromagnetic spectrum is roughly divided into a number of wavelength intervals called bands. The infrared spectral band, of interest within the present context, is generally sub-divided into four lesser bands with arbitrarily chosen boundaries: near infrared $(0.75-3 \mu \mathrm{m})$, middle infrared $(3-6 \mu \mathrm{m})$, far or long infrared $(6-15 \mu \mathrm{m})$ and extreme infrared $(15-1,000 \mu \mathrm{m})$. Most currently used IR camera detectors are sensitive in the middle (MWIR) and the long (LWIR) spectral bands, but the band between $\sim 5$ and $\sim 7.5 \mu \mathrm{m}$ is seldom used because of its rather high atmospheric absorption. Detectors are also available in the near infrared (NIR), sometimes called short wavelength (SWIR), and in the extreme infrared bands, but they are not generally used for thermo-fluid-dynamic applications.

By deriving and integrating with respect to $\lambda$, Planck's law leads, respectively, to the following two other laws:

\subsection{Wien's displacement law}

The wavelength $\lambda^{*}$ at which the black body emits its maximum spectral emissive power is a function of the absolute black body temperature according to:

$\lambda^{*} T=2,897.8 \mu \mathrm{m}-K$

i.e., the maximum value of $I^{b}$ moves toward shorter wavelengths as the body temperature increases. E.g., at liquid nitrogen temperature $(77 \mathrm{~K}) \lambda^{*} \approx 38 \mu \mathrm{m}$, at room temperature $\lambda^{*} \approx 10 \mu \mathrm{m}$, while the sun (at about $6,000 \mathrm{~K}$ ) emits yellow light peaking around $0.5 \mu \mathrm{m}$ in the visible light spectrum. This explains why IR cameras mainly operate in MWIR and LWIR bands.

\subsection{Stefan-Boltzmann's law}

The total (over all wavelengths) hemispherical emissive power $E^{b}\left(\mathrm{~W} / \mathrm{m}^{2}\right)$ also depends on the absolute black body temperature alone, according to:

$E^{b}=\sigma T^{4}$

where $\sigma$ is the Stefan-Boltzmann's constant, which is equal to $5.6704 \times 10^{-8} \mathrm{~W} / \mathrm{m}^{2} \mathrm{~K}^{4}$. However, since infrared camera detectors capture only a limited band of the whole electromagnetic spectrum, while making measurements with IR thermography, Planck's law (2), rather than Stefan-Boltzmann's law (4), has to be applied.

Real objects almost never comply with the abovedescribed laws even if they may approach the black body behavior in certain spectral bands and conditions. A real object generally emits only a fraction $I(\lambda)$ of the radiation emitted by the black body $I^{b}(\lambda)$ at the same temperature and wavelength. For the sake of ease, $I(\lambda)$ is always referred to the spectral hemispherical emissive power. By introducing the spectral emissivity coefficient, defined as:

$\varepsilon(\lambda)=I(\lambda) / I^{b}(\lambda)$.

Equation (2) can be rewritten for real bodies by simply multiplying its second term by $\varepsilon(\lambda)$ :

$I(\lambda)=\varepsilon(\lambda) \frac{C_{1}}{\lambda^{5}\left(e^{C_{2} / \lambda T}-1\right)}$.

Bodies having their emissivity independent of $\lambda$ are called gray bodies. Kirchhoff's law states that the spectral emissivity coefficient is equal to the spectral absorptivity coefficient $\alpha_{r}(\lambda)$, which is the absorbed fraction of the radiation of wavelength $\lambda$. Therefore, for non-transparent bodies, such as those generally used in infrared thermography, Eq. (1) becomes:

$\varepsilon(\lambda)+\rho_{\mathrm{r}}(\lambda)=1$.

Therefore, materials with low emissivity $\varepsilon$ (such as metallic ones) not only emit less energy but also reflect a large amount of the radiation coming from the ambient and impinging on them. Whenever possible, they should not be employed in IR thermography or, if they must be necessarily used and transient heat transfer is not involved, the viewed body should be covered with a thin layer of thermally black paint (such as white dull enamel).

Besides, real objects almost never emit in a diffuse (isotropic) way, the emissivity coefficient $\varepsilon$ being dependent also on the angle $\theta$ (directional emissivity) between the direction of emission and the normal to the emitting surface (viewing angle). The directional emissivity behavior of dielectric materials is quite different from metallic ones.

As shown in Fig. 1 for Macor ${ }^{\mathrm{TM}}$, dielectric materials, at angles $\theta$ generally less than about $60^{\circ}$, have a quite constant and relatively high emissivity, while they emit poorly at large angles away from normal direction. Vice versa, electric conductors (such as platinum) have usually a low emissivity, with a relative minimum in the normal direction. To evidence viewing angle effects, the thermogram of the wall of a hot vertical PVC (dielectric) cylinder under natural convection conditions is shown in Fig. 2. Wall temperature, which is constant cross-wise (along the broken line), appears to decrease toward the cylinder edges, 


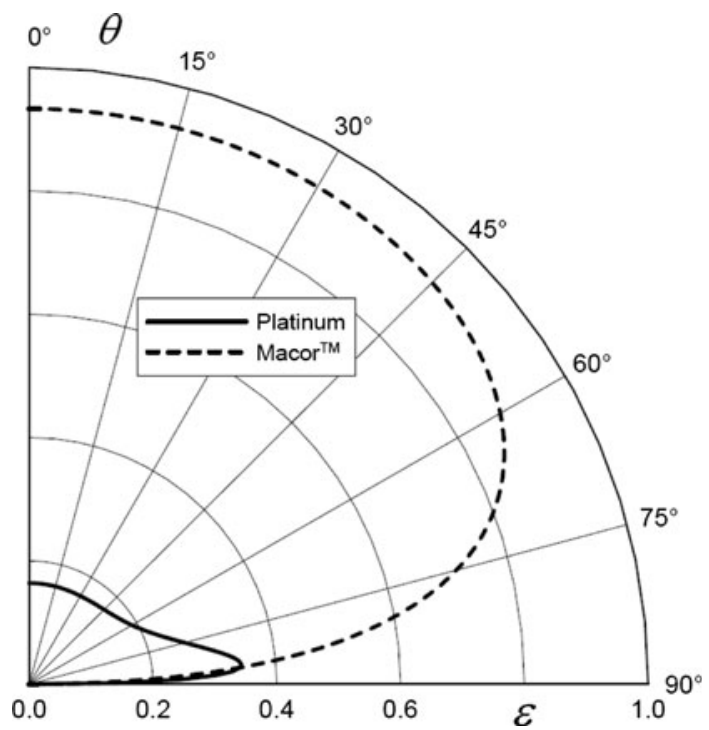

Fig. 1 Emissivity coefficient $\varepsilon$ for $\operatorname{Macor}^{\mathrm{TM}}(8-12 \mu \mathrm{m})$ and platinum $(2 \mu \mathrm{m})$ as a function of viewing angle $\theta$

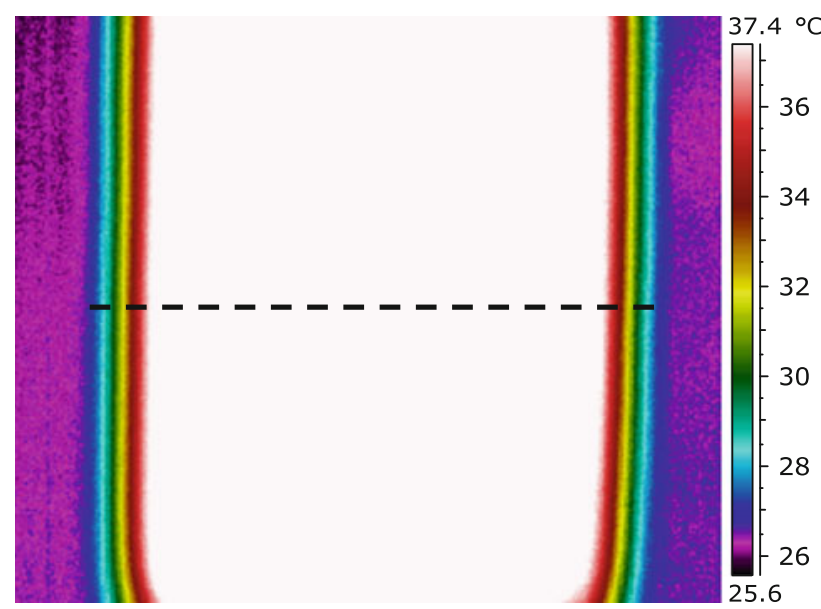

Fig. 2 Temperature map of a vertical PVC cylinder under natural convection

due to high viewing angle and dropping emissivity there. Since infrared measurements are often performed with dielectric materials at low $\theta$ values and because of the limited wavelength band captured by camera detectors, the emissivity of viewed surface is often assumed to be a constant. Being necessary to work at high $\theta$ 's, a careful calibration for $\varepsilon$ as a function of $\theta$ must be performed and viewing angles have to be very well known.

\section{Infrared cameras}

\subsection{Camera hardware}

The core of the IR camera system is the radiation detector. Detector technologies are mainly separated into two

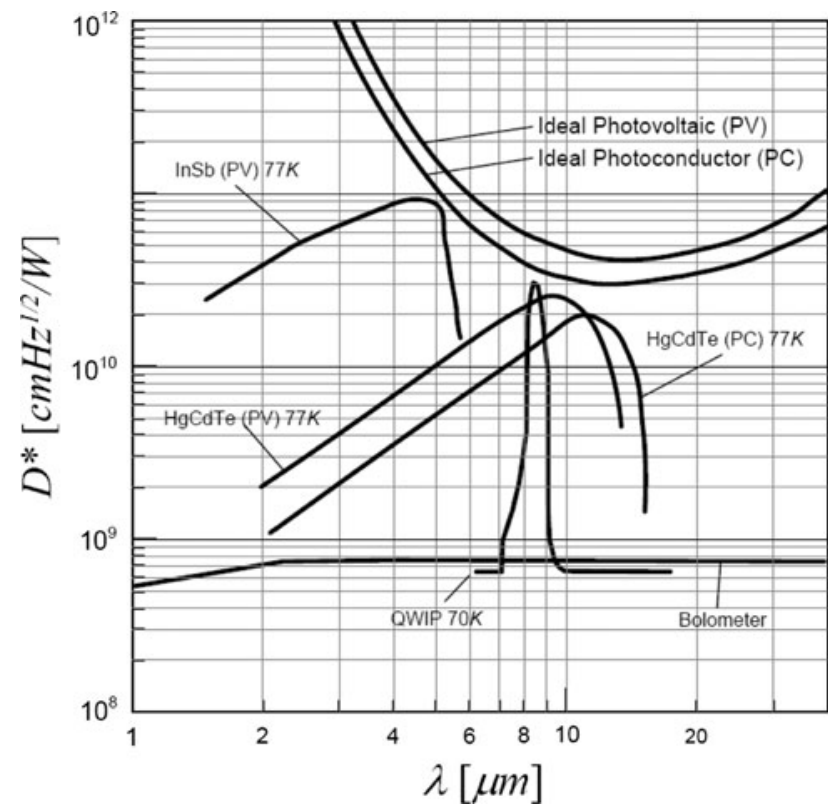

Fig. 3 Spectral responses of some infrared detectors and relative detectivity $D^{*}$

classes: thermal detectors, which are sensitive to the incident energy flux and quantum detectors, whose sensitivity depends on photon absorption.

A currently common type of thermal detector is the uncooled microbolometer, made of metal compound or semiconductor material. The microbolometer typically has a lower cost and broadband IR spectral response but is less sensitive and quite slower (response time in the milliseconds domain) than quantum detectors. The latter ones are made from materials such as InSb, HgCdTe (MCT), and layered GaAs/AlGaAs for QWIP (Quantum Well Infrared Photon) detector. Quantum detectors have a low response time (microseconds domain) and higher sensitivity than thermal ones but their strong cooling is still required. In actual cameras, miniature Stirling coolers easily achieve temperatures around $70 \mathrm{~K}$.

In Fig. 3, the spectral responses of some of most sensitive, largely used, cooled and uncooled infrared detectors and their relative detectivity are indicated. The response curves are shown in terms of $D^{*}$ (detectivity star) for a specific exposure time, as a function of the spectral wavelength. $D^{*}$ is the sensitivity figure of merit of the infrared detector detectivity, so that higher $D^{*} s$ indicate better performance. Modern quantum detectors and QWIP tend to approach the ideal (top curves) detector behavior.

Nowadays, non-cooled microbolometers are typically employed in both MWIR and LWIR spectral bands. Instead, because of their, respectively, higher $D^{*} s$ (see Fig. 3), quantum detectors usually utilize InSb in MWIR band and $\mathrm{HgCdTe}$, or QWIP, in LWIR band. 
To carry out IR thermography, the choice of the most appropriate working spectral band (MWIR, or LWIR) depends on several factors often linked to object surface nature and to atmospheric absorption. Cameras in LWIR band, because of their band low atmospheric absorption (except for very high water vapor content) and high thermal contrast, generally allow for high accuracy measurements; in particular they are able to take data also in the presence of air plasma that typically does not emit in 8-12 $\mu \mathrm{m}$ band (Cardone 2007). However, frequently MWIR cameras are preferred because of their lower cost detectors and optics, higher detectivity $D^{*}$ for quantum detectors and since some surfaces have higher emissivity coefficients in the middle infrared band. It has to be noted that, for temperatures higher than about $10^{\circ} \mathrm{C}$, temperature sensitivity is generally better for MWIR than for LWIR quantum detectors. Should high sensitivity and very short response time not be required, the best and cheapest actual choice is a camera with non-cooled microbolometers.

The standard instantaneous output of each thermal image is generally constituted by a matrix of data from $100 \mathrm{k}$ up to $1 \mathrm{M}$ elements. Therefore, as already stated, a digital data processing is compulsory, which is easy to perform because practically all cameras have a digital output. Temperature range that can be usually measured spans from -20 up to $+1,500^{\circ} \mathrm{C}$, but it can be further extended to higher values by filtering the oncoming radiation. Typically, MWIR cameras use silicon optics, while LWIR ones germanium optics. Most of the available IR cameras offer also telescopes, expander lenses and supplementary close-up microscope lenses, i.e., the possibility of changing their optics, so as to adapt working distances to the scene one is looking at.

\subsection{Camera performance}

The performance of an infrared camera is conventionally evaluated in terms of thermal sensitivity, scan speed, image resolution and intensity resolution.

Thermal sensitivity is generally defined in terms of (mean) noise equivalent temperature difference (NETD), which is the time standard deviation averaged over all pixels of a blackbody scene and is expressed in terms of $\mathrm{mK}$. Some modern FPA systems are able to detect temperature differences of less than $20 \mathrm{mK}$ at ambient temperature.

The scan speed represents the rate at which a complete image (frame) is updated and is expressed in terms of frame rate $(\mathrm{Hz})$. FPA systems of the last generation are characterized by high standard frame rates (often greater than $100 \mathrm{~Hz}$ ) and reducing the number of acquired pixels (i.e., by windowing the frame) and their integration time (i.e., detector sensitivity), frame rates can currently go up to several ten thousands of $\mathrm{Hz}$.

The image resolution, which is the capability of a system to measure surface temperature of small objects, is usually defined as instantaneous field of view (IFOV), which is expressed in terms of mrad; theoretically, IFOV is the ratio of detector pixel width over lens focal length. However, this is only an ideal design parameter and does not fully describe the performance of the actual system. In fact, when a lens of reduced total field of view (FOV) is used, IFOV decreases, but the number of IFOVs in the frame remains the same. Then, the number of IFOVs (pixels) that describes the complete FOV gives a better description of the image resolution. Modern systems are able to produce images composed of up to several hundred thousands of pixels at standard frame rates. More rigorously, the spatial resolution of the IR camera may be defined in terms of the Modulation Transfer Function $\left(\mathrm{MTF}_{\mathrm{IR}}\right)$, which is the mathematical description of the detected signal modulation (also due to the pixel finiteness) as a function of the spatial frequency. This point is further addressed in Sect. 5.2.

The intensity resolution, or dynamic range, is expressed in terms of the number of digital intensity levels the thermal image is composed of. The latest generation of cameras provides typically 14-bit A/D conversion, which allows measuring small temperature differences even in the presence of a large temperature range in the viewed scene.

\subsection{Camera calibration}

The energy actually detected by an IR system depends not only on the emissivity coefficient of the surface under measurement but also on environmental conditions. In fact, the total radiation $I^{\prime}$ detected by the camera can be written as:

$I^{\prime}=\tau_{\mathrm{r}} \varepsilon I_{\mathrm{obj}}^{b}+\tau_{\mathrm{r}}(1-\varepsilon) I_{\mathrm{amb}}^{b}+\left(1-\tau_{\mathrm{r}}\right) I_{\mathrm{atm}}^{b}$,

where $\tau_{r}$ is the transmissivity of the atmosphere between the surface and the camera, $I_{\mathrm{obj}}^{b}$ is the radiation intensity corresponding to a black body at the object temperature $T_{\mathrm{obj}}, I_{\mathrm{amb}}^{b}$ is the black body radiation intensity corresponding to the (effective) temperature of the object surroundings $T_{\mathrm{amb}}$, called the reflected ambient (or background) temperature and $I_{\mathrm{atm}}^{b}$ is the radiation intensity corresponding to a black body at the temperature of the atmosphere $T_{\mathrm{atm}}$. In Eq. (8), the term $\tau_{r} \varepsilon I_{\mathrm{obj}}^{b}$ is the emission from the surface captured by the radiometer, $\tau_{r}(1-\varepsilon)$ $I_{\mathrm{amb}}^{b}$ is the reflected emission from ambient sources, and $(1-\varepsilon)$ is the surface reflectivity $\left(T_{\mathrm{amb}}\right.$ is assumed constant for all emitting surfaces of the half sphere seen from the object surface). The term $\left(1-\tau_{r}\right) I_{\mathrm{atm}}^{b}$ is the emission from 
the atmosphere, with $\left(1-\tau_{r}\right)$ being the atmosphere emissivity. For the sake of simplicity in the Eq. (8), the dependence from $T$ and wavelength is not indicated.

Generally, the transmissivity of the atmosphere $\tau_{r}$ is assumed to be equal to unity, which simplifies Eq. (8). Thus, using Eqs. (2) and (8), a semi-empirical adaptation of Planck's law with the parameters $R, B$, and $F$ can be found. The typical calibration function of an IR camera can be put in the form:

$U_{\mathrm{D}}=\varepsilon \frac{R}{e^{B / T_{\mathrm{obj}}}-F}+(1-\varepsilon) \frac{R}{e^{B / T_{\mathrm{amb}}}-F}$,

where $U_{\mathrm{D}}$ is the detected signal, $R$ is a function of integration time and wavelength, $B$ is a function of wavelength alone, and $F$ is a positive value very close to 1 . If the object used for calibration is a black body, the calibration constants $R, B$, and $F$, besides taking into account the sensed wavelength band and the radiation constants, consider only emission and absorption from atmosphere.

A simpler way to perform the calibration, which does not take in account $T_{\mathrm{amb}}$ variations, is the in situ calibration proposed by Sargent et al. (1998), which practically neglects the second term of the second member of Eq. (9).

Needless to say that, if a window exists in the optical path between test section and IR camera (as may happen in wind tunnel measurements, e.g., see Fig. 11), the constants of Eq. (9) depend also on the transmissivity through such a window.

\section{Heat flux sensors}

The convective heat flux $Q_{\mathrm{c}}$ (energy rate per unit area, $\mathrm{W} / \mathrm{m}^{2}$ ) between a fluid and a surface at temperature $T_{\mathrm{w}}$ is governed by Newton's law:

$Q_{\mathrm{c}}=h\left(T_{\mathrm{w}}-T_{\mathrm{r}}\right)$

$h$ being the convective heat transfer coefficient $\left[\mathrm{W} /\left(\mathrm{m}^{2} \mathrm{~K}\right)\right]$ and $T_{r}$ a reference temperature of the fluid. The reference temperature depends on the stream experimental conditions, for example, for external low Mach number flows, the reference temperature practically coincides with the undisturbed stream one. Instead, for high Mach number flows (Shapiro 1954) or mixing of two streams at different temperatures, such as warm jets in ambient air, the correct choice is the adiabatic wall temperature $T_{\text {aw }}$.

The present main interest is to measure either $Q_{\mathrm{c}}$, or $h$, but it has to be noted that as in the case of film cooling (see Sect. 6.2), IR thermography practically allows the measurement of $T_{\text {aw }}$ itself so as to determine surface adiabatic effectiveness maps.

Data are generally presented in terms of either Nusselt number $N u=h l / k$ (especially for internal flows) or Stanton number $S t=h /\left(\rho c_{\mathrm{p}} V\right)$ (external flows), where $l$ is a characteristic length of the problem and $k, \rho c_{\mathrm{p}}$ and $V$ are, respectively, the thermal conductivity coefficient, the specific heat per unit volume at constant pressure and a characteristic velocity of the fluid medium.

Heat-flux sensors of interest herein generally consist of plane slabs, with a known thermal behavior, whose temperature has to be measured at set points. The energy conservation equation applied to the proper sensor model yields the relationship by which measured temperatures are correlated to heat flux. In practice, the slab can also be curved, its curvature in such a case being ignored only as long as the layer affected by the exchanged heat flux is small when compared to the slab local curvature radius. The sensor is often supposed ideal, which means that its material thermo-physical properties are independent of temperature. The slab surface the flow is going over is called front surface, while the opposite one, back surface.

The simplest model of a sensor is the one-dimensional one, where heat flux to be measured is supposed to be normal to the sensing element surface, i.e. the temperature gradient components parallel to the slab surfaces are assumed negligible. In this section, one-dimensional sensors are considered, their operation being extended to the multi-dimensional case in Sect. 5.

The heat flux sensors mainly used with IR thermography are as follows:

1. Heated-thin-foil This sensor consists of a thermally thin metallic foil, or a printed circuit board, steadily and uniformly heated by Joule effect. Foil heating can be accomplished also in a different way, e.g., by a known steady radiation input to foil. Measuring sensor surface temperature and performing an energy balance allows computing heat flux. In this sensor, due to foil thermal thinness, either one of its surfaces can be generally viewed by IR camera. An extension to foils not thermally thin can be performed.

2. Thin-film Classically, a very thin resistance thermometer (film) measures the surface temperature of a thermally thick slab to which is bonded. Heat flux is inferred from the theory of heat conduction in a semiinfinite solid. The surface film must be very thin so as to have negligible heat capacity and thermal resistance as compared to the layer affected by the exchanged heat flux. When using this sensor with IR thermography, the film does not exist and the heat-exchanging surface must be necessarily viewed by camera. As such, fluids at least partially transparent $\left(\tau_{r} \neq 0\right)$ in the used IR band must be employed.

3. Wall calorimeter or thin-skin The slab is made thermally thin (so that its temperature can be assumed 
to be constant across its thickness) and is used as a simple calorimeter. Heat flux is computed from the time rate of the slab temperature change, usually measured by a thermocouple. In the use of this sensor with IR thermography, the skin can be made really thin, because there are no thermocouples, and either one of the slab surfaces can be generally viewed by the infrared camera.

A detailed analysis of the heat flux sensors that can be used with IR thermography can be found in Carlomagno and de Luca (1989) and Astarita et al. (2006).

Recently, heat flux sensors, based on a numerical solution of Fourier's law (typically described by an inverse heat transfer model) and surface temperature measurements, have been developed (see acronym NIHT in Table 1), e.g., to take into account temperature dependence of thermophysical properties and/or to have slabs with high curvature, but, for the sake of brevity, they will not be described herein.

Thin-film and wall calorimeter sensors belong to the class of transient techniques, while heated-thin-foil sensor represents a steady technique. Transient techniques generally regard passive heating due to some existing temperature difference between sensor surface and flow, while heated-thin-foil involves active heating.

When using IR thermography, transient techniques should be mainly applied to experimentally analyze the constant convective heat transfer coefficient boundary condition, while the heated-thin-foil steady technique is functional to the constant heat flux one. A constant temperature boundary condition can be implemented with transient techniques, large temperature differences between sensor and flow and relatively short measuring times.

\section{Analysis of measurement errors}

The problem of restoring in a general sense the recorded thermal images is very important from both the imaging and the sensor points of view. E.g., as shown by de Luca et al. (1990a, 1993) and Simeonides et al. (1993) in the study of Goertler vortices in hypersonic flow, the requirement of data restoration is stressed in heat transfer phenomena where relatively high temperature gradients are present. In fact, the possibility that accurate measurements of variable convective heat transfer coefficients may be performed by means of IR thermography depends on the chance that all potential error sources, linked to environment, thermal sensor and IR camera, can be removed. With regard to errors due to the environment, they can be automatically corrected by performing an accurate calibration as indicated in Sect. 3 .

\subsection{Errors due to the thermal sensor}

Errors due to sensor are mainly associated with radiation that it emits toward ambient, tangential conduction within the sensor itself and losses from its back surface, as illustrated in Fig. 4. When standard techniques are used to measure wall temperature, it is possible to have a very low surface emissivity coefficient (e.g., by gold plating the sensor surface) so as to neglect the radiative heat flux to ambient. Obviously, because of what is stated in Sect. 2, this cannot be the case when using an IR camera, since surface emissivity should be high.

Radiative heat flux is computed with the so-called radiosity law which derives from Eq. (4):

$Q_{\mathrm{r}}=\sigma \varepsilon_{t}\left(T_{\mathrm{w}}^{4}-T_{\mathrm{amb}}^{4}\right)$

where $\varepsilon_{t}$ is the front surface total hemispherical emissivity coefficient; $T_{\mathrm{w}}$ and $T_{\mathrm{amb}}$ are the temperature of the sensor surface and of the experimental ambient, respectively.

As far as sensor errors due to tangential conduction, the assumption of zero-dimensional sensor is strictly satisfied only if the sensor temperature is constant along sensor surface (tangential) direction. However, with two-dimensional IR thermography, one is interested in studying complex thermo-fluid-dynamic phenomena where sensor surface temperature generally varies. Temperature variations inevitably cause conductive heat fluxes in tangential direction, which may constitute an important part of the exchanged heat.

For the heated-thin-foil sensor and isotropic foils, by retaining the assumption that sensor is thermally thin (i.e. isothermal at temperature $T_{\mathrm{w}}$ across its thickness $s$ ) and ideal, it is possible to evaluate the equivalent tangential conduction heat flux $Q_{k}$ per unit sensor area by means of Fourier's law:

$Q_{k}=-\lambda s \nabla^{2} T_{\mathrm{w}}$

Therefore, to extend the heated-thin-foil sensor to the multi-dimensional case, one must include in the no longer one-dimensional energy balance this contribution as well.

It is important to remark that infrared thermography (intrinsically two-dimensional) allows, in principle, to directly evaluate the Laplacian of Eq. (12) by numerical computation. However, since in the evaluation second derivatives are involved, this can be accomplished only after filtering the camera signal, typically affected by noise (Astarita and Cardone 2000).

As already mentioned, in many applications of the heated-thin-foil sensor, a spatially constant Joule heating can be obtained by using a printed circuit board (Fénot et al. 2008). The printed circuit is generally manufactured with several adjacent thin (down to $5 \mu \mathrm{m}$ ) copper tracks, closely spaced, arranged in a Greek fret mode (see Fig. 5) 
Table 1 Main wall convective heat transfer measurements and surface flow visualization performed by infrared thermography

\begin{tabular}{|c|c|c|c|}
\hline Topic & Authors & Flux sensor & IR detector \\
\hline \multirow[t]{9}{*}{ Film cooling } & Kutscher (1994) & TS & - \\
\hline & Leger et al. (1996) & $\mathrm{T}$ & S/MW \\
\hline & Sargent et al. (1998) & HTF & $\mathrm{S} / \mathrm{LW}$ \\
\hline & $\begin{array}{l}\text { Dizene et al. (2000), Dorignac et al. }(2001,2005) \\
\text { and Petre et al. (2003) }\end{array}$ & $\mathrm{T}, \mathrm{HTF}$ & $\mathrm{S} / \mathrm{LW}$ \\
\hline & Baldauf et al. (2002) & NIHT & - \\
\hline & Ekkad et al. (2004) & $\mathrm{TF}$ & FPA/LW \\
\hline & Lu et al. (2007) and Dhungel et al. (2009) & $\mathrm{TF}$ & $\mathrm{FPA} / \mathrm{B}$ \\
\hline & Waye and Bougard (2007) & $\mathrm{TF}$ & $\mathrm{FPA} / \mathrm{B}$ \\
\hline & Zhang et al. (2009) & $\mathrm{TF}$ & FPA/B \\
\hline \multirow[t]{13}{*}{ Jets } & $\begin{array}{l}\text { Meola et al. (1995,1996), Carlomagno et al. }(2002,2004) \\
\text { and Meola and Carlomagno (2004) }\end{array}$ & HTF & $\mathrm{S} / \mathrm{LW}$ \\
\hline & Abdul Nour et al. (2000) & HTF & S_LW \\
\hline & $\begin{array}{l}\text { Narayanan et al. }(2003,2004 a, b), \text { Narayanan and Patil (2007a) } \\
\text { and Narayanan }(2007 b)\end{array}$ & HTF, T & S/LW \\
\hline & Loubat et al. (2004) & NIHT & $\mathrm{S} / \mathrm{LW}$ \\
\hline & Feldheim et al. (2005) & HTF & $\mathrm{S} / \mathrm{LW}$ \\
\hline & Fenot et al. $(2005,2008)$ & HTF & FPA/MW \\
\hline & Patil and Narayanan $(2005 a, b)$ & HTF & FPA/MW \\
\hline & Goodro et al. $(2007 \mathrm{a}, \mathrm{b})$ & HTF & FPA/MW \\
\hline & Goodro et al. $(2008,2009)$ & HTF & FPA/B \\
\hline & Kim and Giovannini (2007) & HTF & FPA/MW \\
\hline & $\mathrm{Li}$ and Chen (2007) & $\mathrm{T}$ & FPA/B \\
\hline & Roger (2007) & IHT & FPA/MW \\
\hline & Attalla and Specht (2009) & HTF & - \\
\hline \multirow[t]{10}{*}{ Channels and ribs } & Aliaga et al. (1994) & HTF & $\mathrm{S} / \mathrm{LW}$ \\
\hline & Lorenz et al. $(1995,1996)$ & HTF & $\mathrm{S} / \mathrm{LW}$ \\
\hline & $\begin{array}{l}\text { Cardone et al. (1998), Astarita and Cardone (2000, 2003), } \\
\text { Carlomagno et al. (2002) and Astarita et al. (2002a, b, 2006) }\end{array}$ & HTF & $\mathrm{S} / \mathrm{LW}$ \\
\hline & Buchlin (2002) & HTF & $\mathrm{S} / \mathrm{LW}$ \\
\hline & Fedrizzi and Arts (2004) & NIHT & FPA/MW \\
\hline & Won et al. (2004b) & HTF & $\mathrm{S} / \mathrm{LW}$ \\
\hline & Booten and Eaton (2007) & $\mathrm{TF}$ & FPA/MW \\
\hline & Gallo et al. (2007) & HTF & $\mathrm{S} / \mathrm{LW}$ \\
\hline & Dees and Bogard (2008) & HTF & FPA/B \\
\hline & Gupta et al. (2008) & HTF & FPA/B \\
\hline \multirow[t]{6}{*}{ Transition } & de Luca et al. (1990b, 1995a) & HTF & $\mathrm{S} / \mathrm{LW}$ \\
\hline & $\begin{array}{l}\text { Gartenberg and Roberts (1991a, b), Gartenberg et al. (1992) } \\
\text { and Gartenberg and Wright (1994) }\end{array}$ & $\mathrm{T}$ & $\mathrm{S} / \mathrm{LW}$ \\
\hline & $\begin{array}{l}\text { Cardone et al. (1996, 1997b), Astarita et al. (2002) } \\
\text { and Astarita and Cardone (2008) }\end{array}$ & HTF & $\mathrm{S} / \mathrm{LW}$ \\
\hline & Riedel and Sitzmann (2002) & $\mathrm{T}$ & - \\
\hline & Seraudie et al. (2003) & $\mathrm{T}$ & - \\
\hline & Zuccher and Saric (2008) & $\mathrm{T}$ & FPA/LW \\
\hline \multirow[t]{7}{*}{ Micro systems } & Buffone and Sefiane $(2004,2008)$ & $\mathrm{T}$ & FPA/LW \\
\hline & Patil and Narayanan (2006) & HTF & FPA/MW \\
\hline & Boye et al. (2007) and Diaz et al. (2007) & HTF & $\mathrm{S} / \mathrm{LW}$ \\
\hline & Liu et al. $(2007,2009)$ & $\mathrm{T}, \mathrm{HTF}$ & - \\
\hline & Mishan et al. (2007) & HTF & - \\
\hline & Christofferson et al. (2008) & $\mathrm{T}$ & FPA/MW \\
\hline & Veeraragavan and Cadou (2008) & $\mathrm{T}$ & - \\
\hline
\end{tabular}


Table 1 continued

\begin{tabular}{|c|c|c|c|}
\hline Topic & Authors & Flux sensor & IR detector \\
\hline \multirow[t]{5}{*}{ Two phase } & Hetsroni et al. (1998a, b, 2003) and Klein et al. (2005) & HTF & - \\
\hline & Freund et al. (2007) & NIHT & FPA/B \\
\hline & Tarozzi et al. (2007) and Tartarini et al. (2009) & $\mathrm{T}$ & FPA/B \\
\hline & di Francescantonio et al. (2008) & $\mathrm{T}$ & FPA/LW \\
\hline & Jalilvand et al. (2008) & $\mathrm{T}$ & - \\
\hline \multirow[t]{9}{*}{ Flow instabilities } & de Luca et al. (1990a, 1993, 1995b) and Aymer de la Chevalery et al. (1997) & $\mathrm{TF}$ & $\mathrm{S} / \mathrm{LW}$ \\
\hline & Abid et al. (1993) & $\mathrm{T}$ & - \\
\hline & Simeonides et al. (1993) & $\mathrm{TF}$ & $\mathrm{S} / \mathrm{LW}$ \\
\hline & Meola et al. (1995) & HTF & $\mathrm{S} / \mathrm{LW}$ \\
\hline & Sargent et al. (1998) and Hedlund et al. (1999a, b) & HTF & $\mathrm{S} / \mathrm{LW}$ \\
\hline & Hetsroni et al. (2001) and Zacksenhouse et al. (2001) & HTF & - \\
\hline & Inagaki and Okamoto (1999) and Inagaki et al. (2006) & $\mathrm{T}$ & FPA/LW \\
\hline & Narayanan and Patil (2007) and Narayanan (2007) & HTF & $\mathrm{S} / \mathrm{LW}$ \\
\hline & Rahal et al. (2007) & $\mathrm{T}$ & FPA/MW \\
\hline \multirow[t]{6}{*}{ Rotating surfaces } & Cardone et al. (1996, 1997a), Astarita et al. (2002) and Astarita and Cardone (2008) & HTF & $\mathrm{S} / \mathrm{LW}$ \\
\hline & Boutarfa and Harmand (2005) and Pelle and Harmand (2006, 2007a, b, 2008, 2009) & TS & - \\
\hline & Seghir-Ouali et al. (2006) & TS & - \\
\hline & Mori et al. (2007, 2008), Sekavcnik et al. (2008) and Novak et al. (2008) & $\mathrm{T}$ & FPA/SW \\
\hline & Bajcar et al. (2008) & $\mathrm{T}$ & FPA/B \\
\hline & Lyons et al. (2008) & $\mathrm{TF}$ & - \\
\hline \multirow[t]{7}{*}{ Separation/bluff body } & de Luca et al. $(1992,1995 b)$ & $\mathrm{TF}$ & $\mathrm{S} / \mathrm{LW}$ \\
\hline & Cardone et al. (1997b) & HTF & $\mathrm{S} / \mathrm{LW}$ \\
\hline & Meinders et al. (1997, 1999a, b) and Meinders and Hanjalic (2002) & HTF & S/MW \\
\hline & Ghisalberti and Kondjoyan (2001) & HTF & FPA/MW \\
\hline & Montelpare and Ricci (2004a), Ricci and Montelpare $(2005,2006)$ and Ricci et al. (2007) & HTF & FPA/LW \\
\hline & Mai et al. (2008) & $\mathrm{T}$ & - \\
\hline & Menfoukh et al. (2008) & $\mathrm{T}$ & FPA/B \\
\hline \multirow[t]{10}{*}{ Hypersonic flows } & de Luca et al. (1990a, 1992, 1993, 1995b) and Aymer de la Chevalery et al. (1997) & $\mathrm{TF}$ & $\mathrm{S} / \mathrm{LW}$ \\
\hline & Balageas et al. (1991) & $\mathrm{TF}$ & - \\
\hline & Henckels et al. (1993) and Kreins et al. (1996) & $\mathrm{TF}$ & - \\
\hline & Simeonides et al. (1993) & $\mathrm{TF}$ & $\mathrm{S} / \mathrm{LW}$ \\
\hline & Hozumi (1998) & $\mathrm{TF}$ & - \\
\hline & Le Sant et al. (2002) & NIHT & $\mathrm{S} / \mathrm{LW}$ \\
\hline & Schrijer et al. (2006) & $\mathrm{TF}$ & $\mathrm{S} / \mathrm{LW}$ \\
\hline & Schulein (2006) & $\mathrm{TF}$ & FPA/B \\
\hline & Cardone (2007) & NIHT & FPA/LW \\
\hline & Gulhan et al. (2008) & $\mathrm{TF}$ & FPA/LW \\
\hline \multirow[t]{12}{*}{ Miscellanea } & Carlomagno and de Luca (1989), Astarita et al. (2000) and Carlomagno $(2006,2007)$ & TF, HTF & $\mathrm{S} / \mathrm{LW}$ \\
\hline & Bougeard et al. $(1994,1995)$ and Bougeard (2007) & TS & $\mathrm{S} / \mathrm{LW}$ \\
\hline & Henry et al. (1995) & HTF & - \\
\hline & Watel et al. $(1995,1999,2000 \mathrm{a}, \mathrm{b})$ & TS & $\mathrm{S} / \mathrm{LW}$ \\
\hline & Aydin (1997) & HTF & S/MW \\
\hline & Buchlin (1998) & HTF & $\mathrm{S} / \mathrm{LW}$ \\
\hline & Ligrani et al. (2001), Mahmood and Ligrani (2002), Burgess et al. (2003) and Won et al. (2004a) & HTF & $\mathrm{S} / \mathrm{LW}$ \\
\hline & Ay et al. (2002) & HTF & FPA/MW \\
\hline & Montelpare and Ricci (2004b) and Ricci and Montelpare (2006) & HTF & S/MW \\
\hline & Biniwale et al. (2006) & TS & FPA/MW \\
\hline & Yuki et al. (2008) & $\mathrm{T}$ & FPA/MW \\
\hline & Reulet et al. (2009) & HTF & FPA/MW \\
\hline
\end{tabular}

$T$ temperature, $T F$ thin-film, $T S$ thin-skin, $H T F$ heated-thin-foil, $N I H T$ numerical inverse heat transfer, $S$ infrared scanning camera, $F P A$ infrared focal plane array camera, $B$ microbolometer, $M W$ IR middle wave band, $L W$ IR long wave band 


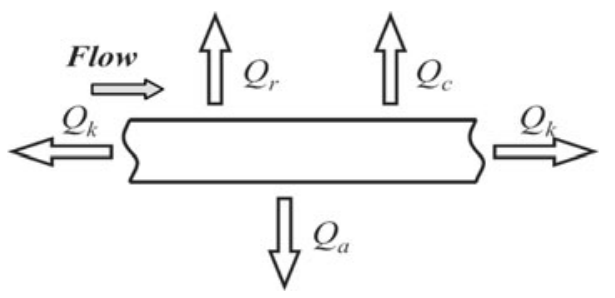

Fig. 4 Thermal sensor with tangential conduction

and bound to a fiberglass substrate. However, due to the high conductivity coefficient of copper, the board exhibits an anisotropic thermal conductance behavior (along, or across, the tracks) so that it is not correct to evaluate $Q_{k}$ by means of (12).

By still retaining the assumption that $T_{\mathrm{w}}$ is independent of coordinate $z$ normal to the board, it is, therefore, necessary to generalize Eq. (12) to take into account this effect:

$Q_{k}(x, y)=-\underline{\nabla} \cdot\left(s(x, y) \underline{\underline{\Lambda}}(x, y) \cdot \underline{\nabla} T_{\mathrm{w}}(x, y)\right)$

where $\Lambda$ is the thermal conductivity tensor. To simplify Eq. (13) and by neglecting the top and bottom edges of the board, it is feasible to roughly separate the effect due to copper tracks from that of fiberglass support. In particular, by choosing a Cartesian coordinate system with its axes directed along the two principal axes $x$ and $y$ of $\underline{\Lambda}$ (see Fig. 5), one may split the effects into two directions, normal to tracks $x$ and parallel to them $y$. This problem is addressed by Astarita and Cardone (2000).

Also for both thin-skin and thin-film sensors, the temperature within the sensor was supposed to be one-

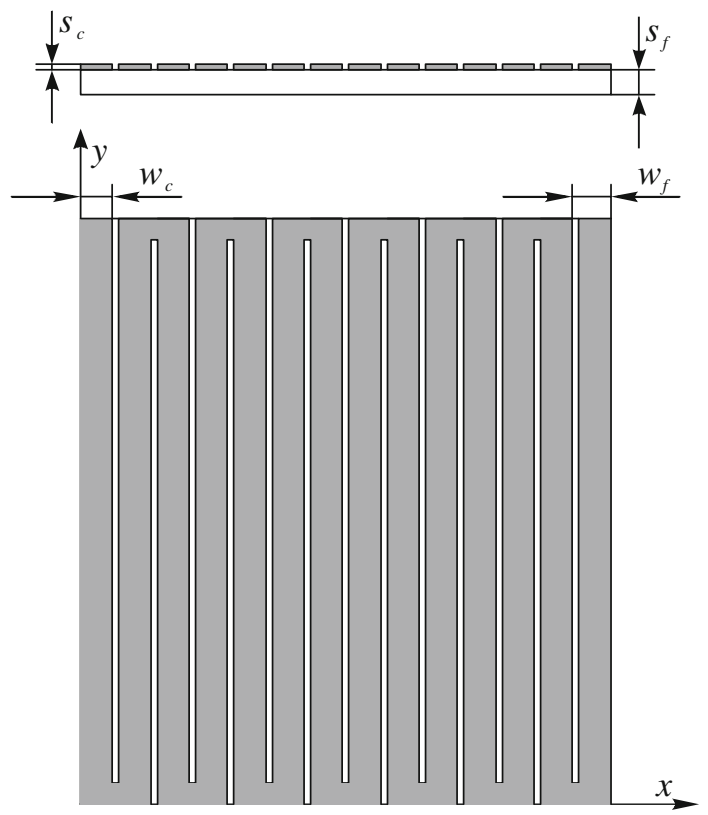

Fig. 5 Heated-thin-foil sensor made with a printed circuit board dimensional. Under the assumption that the sensor material is isotropic, or (as already done before) by choosing a Cartesian coordinate system with its axes directed as the two principal axes of the thermal conductivity tensor, it is possible to split conduction effects along the two tangential directions. Therefore, since the extension to any arbitrary convective heat flux is straightforward, for the sake of ease, in the following it is assumed that convective heat flux harmonically varies only along one direction parallel to the sensor surface $x$, that is:

$Q_{\mathrm{c}}(x)=Q_{\mathrm{h}} \cos (\xi x)$

where $Q_{h}$ is the heat flux amplitude and $\xi=2 \pi / L$ the wave number ( $L$ being the wavelength).

To correct measured temperatures so as to take into account tangential conduction effects, it is convenient to use the ratio between the temperature amplitudes with and without tangential conduction Temperature Amplitude Transfer Function $(F)$, which, according to De Luca and Cardone (1991), is reported in Fig. 6, for the two sensors as a function of a modified Fourier number $\mathrm{Fo}_{\xi}=\xi^{2} / \alpha t$, where $\alpha$ is the thermal diffusivity coefficient of sensor material.

The amplitude of each harmonic component of the measured temperature may be thus corrected and the corresponding harmonic component of the heat flux can be evaluated by using the classical one-dimensional formulae. A direct comparison between the two sensors has practically no meaning because they are basically realized with materials having different thermal conductivities (high for thin-skin and low for thin-film). Nonetheless, it has to be noted that, for the thin-skin sensor, the tangential conduction correction can be directly computed as in the case of the heated-thin-foil with Eq. (12), as a function of time.

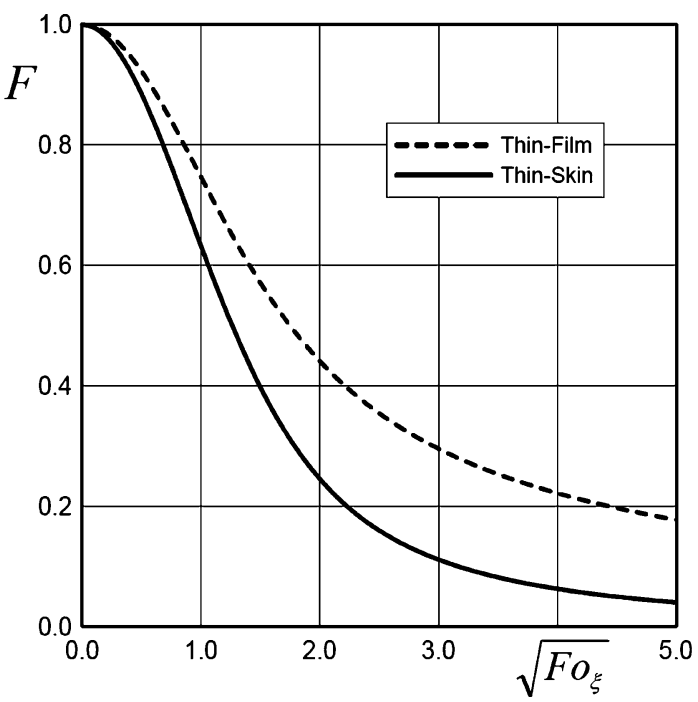

Fig. 6 Temperature amplitude transfer function for unsteady sensors 
If back surface is diabatic as indicated in Fig. 4, data reduction has to be performed by subtracting to $Q_{j}$ also the total heat flux from sensor to external ambient $Q_{\mathrm{a}}$, via the back surface. Typically, this heat flux results to be the sum of radiative and natural convection heat fluxes. Radiative heat flux can again be computed by means of radiosity law (11), while convective heat flux to external ambient may be evaluated by using standard correlations tables. However, to carefully evaluate $Q_{\mathrm{a}}$, it is much better to perform some ad hoc tests with thermally insulated front surface and which include the radiative contribution as well (Astarita and Cardone 2000).

\subsection{Errors due to the camera}

The relation between the original thermal object $o(x, y)$ and the recorded degraded image $r(x, y)$ is given by the twodimensional point spread function $\operatorname{PSF}(x, y)$, where $x$ and $y$ are the spatial coordinates. Function PSF describes the response of the measuring process to a point source (or point object). A general expression for $r(x, y)$ is:

$r(x, y)=\operatorname{PSF}(x, y) \otimes o(x, y)+n(x, y)$

where $\otimes$ is the convolution operator and $n(x, y)$ indicates an additional noise term. If it is assumed that the main subsystems of the whole measurement process, thermal sensor and IR camera (which includes optics, detector and electronics) are shift invariant, PSF may be expressed as:

$\operatorname{PSF}(x, y)=h_{\mathrm{TS}}(x, y) \otimes h_{\mathrm{IR}}(x, y)$

where $h_{\mathrm{TS}}(x, y)$ is the impulse response of thermal sensor and $h_{\mathrm{IR}}(x, y)$, the one of IR camera. After Fourier transform, the convolution product of Eq. (15), in the frequency domain $\zeta_{x}, \zeta_{y}$, reduces to an ordinary product:

$R\left(\zeta_{x}, \zeta_{y}\right)=\operatorname{STF}\left(\zeta_{x}, \zeta_{y}\right) \cdot O\left(\zeta_{x}, \zeta_{y}\right)+N\left(\zeta_{x}, \zeta_{y}\right)$

where STF is the System Transfer Function, which expresses the overall degradation.

In the presence of imaging degradations only, STF coincides with the Optical Transfer Function, which normalized magnitude (with unit value at zero frequency) is generally defined as the Modulation Transfer Function $\left(\mathrm{MTF}_{\mathrm{IR}}\right)$ of the camera. A discussion on the $\mathrm{MTF}_{\mathrm{IR}}$ of IR scanning systems is reported by de Luca and Cardone (1991) and of modern FPA systems by Boreman (2001). Instead, in the most general case, under the assumption that the different modulation processes are cascaded, the product of the various transfer function yields the STF. E. g., for thin-film and thin-skin (see Sect. 5.1) the STF is equal to $\mathrm{MTF}_{\mathrm{IR}} \times F$.

It has to be recalled that, for the heated-thin-foil and thin-skin sensors, the sensor modulation (heat flux losses due to tangential conduction and radiation) may be directly appraised as already indicated (see Sect. 5.1). It is important to point out that $\mathrm{MTF}_{\mathrm{IR}}$ of an IR camera is a property of the entire system. In fact, all components (lens assembly, FPA, A/D converter, cabling, etc.) contribute to the final $\mathrm{MTF}_{\mathrm{IR}}$ of the system. Assuming that all subsystem are cascaded, the system $\mathrm{MTF}_{\mathrm{IR}}$ is given by: $\mathrm{MTF}_{\mathrm{IR}}=$ $\mathrm{MTF}_{\text {Optics }} \cdot \mathrm{MTF}_{\mathrm{FPA}} \cdot \mathrm{MTF}_{\text {Electronics }}$.

In Fig. 7, the measured $\mathrm{MTF}_{\mathrm{IR}}$ of an IR camera, based on 1,024 × 1,024 square pixels MWIR QWIP (Gunapala et al. 2005), is shown as a function of the spatial frequency $\xi$ normalized with respect to Nyquist frequency $\xi_{\mathrm{N}}(1 / 2$ pixel pitch). The MTF $\mathrm{Mptics}_{\text {of }}$ of the spot scanner optics at $\xi_{\mathrm{N}}$ is 0.2 , thus the $\mathrm{MTF}_{\mathrm{FPA}}$ at Nyquist frequency should be around 30 and $45 \%$ along horizontal and vertical direction, respectively. The MTF $_{I R}$ difference along horizontal and vertical directions is also present in the differently shaped PSFs (not shown herein). Since MTF Optics $_{\text {data do not show }}$ a large variation between horizontal and vertical directions, the difference is most probably due to the electronic readout integrated circuit.

\subsection{Image restoration}

From Eq. (17), the degraded image can be restored with a restoration (inverse) filter $W\left(\xi_{x}, \xi_{y}\right)$, which is the reciprocal of STF, yielding the restored image $\tilde{o}(x, y)$ :

$\tilde{o}(x, y)=F^{-1}\left(W\left(\xi_{x}, \xi_{y}\right) R\left(\xi_{x}, \xi_{y}\right)\right)$

where $F^{-1}$ indicates the inverse Fourier transform. Restoration with the inverse filter $W\left(\xi_{x}, \xi_{y}\right)$ results in unlimited amplification of the response when modulation approach zero. This undesired amplification can be prevented by stopping the restoration process just before the noise frequencies or using the Wiener filter $W_{\mathrm{w}}\left(\xi_{x}, \xi_{y}\right)$ according to Jain (1989):

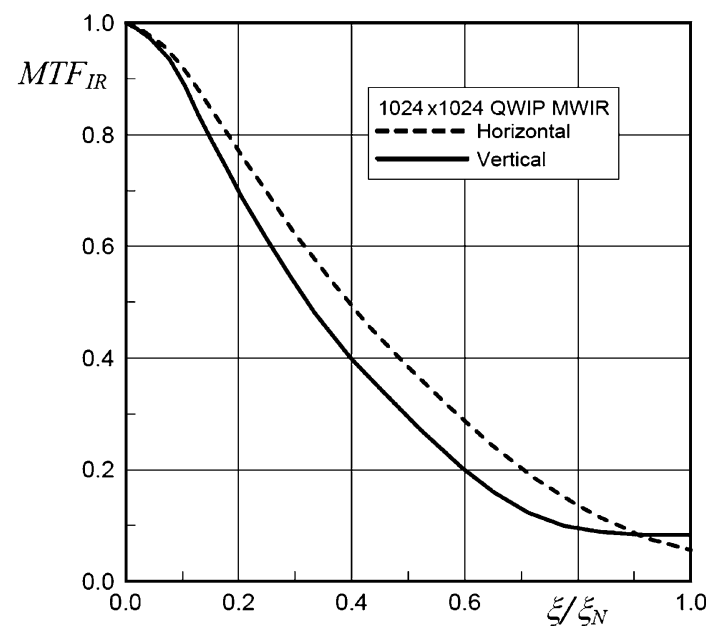

Fig. 7 Measured $\mathrm{MTF}_{\mathrm{IR}}$ of an IR Camera. From Gunapala et al. (2005) 
$W_{w}\left(\xi_{x}, \xi_{y}\right)=\frac{\operatorname{STF}^{*}\left(\xi_{x}, \xi_{y}\right)}{\left|\operatorname{STF}^{*}\left(\xi_{x}, \xi_{y}\right)\right|^{2}+\Phi_{\text {noise }} / \Phi_{\text {image }}}$

where $\operatorname{STF}^{*}\left(\xi_{\mathrm{x}}, \xi_{\mathrm{y}}\right)$ is the complex conjugate of the STF and $\Phi_{\text {noise }}$ and $\Phi_{\text {image }}$ are the power spectral densities of noise and image, respectively. The additional ratio $\Phi_{\text {noise }} / \Phi_{\text {image }}$ (image signal to noise ratio) attenuates high noise components. The Wiener filter becomes the exact inverse filter for noise-free images.

An example of image restoration for the thin-skin sensor is presented by Bougeard (2007) who studies the local heat transfer on a plate fin which had two-tube rows normal to it. Heat transfer measurements are corrected for radiation and tangential conduction effects. The fin is warmed up to $42^{\circ} \mathrm{C}$ and then cooled down by airflow with a fixed entrance temperature of $22^{\circ} \mathrm{C}$. The IR camera records temperature variations during a 6.5-s duration (about one hundred thermal images). In order to reduce noise, Bougeard computes the integral (between initial and final time of test) of the terms which are present for the thin-film sensor and evaluates the tangential conduction with Eq. (12).

By indicating with $e_{\text {tot }}$, the integral term corresponding to the total internal energy per unit area lost by the fin $\left(\mathrm{J} / \mathrm{m}^{2}\right), e_{\text {cond }}$ the integral of the conductive term, $e_{\text {rad }}$ the integral of the radiative term, and $i_{\text {temp }}$ the term corresponding to the integration of the difference of fin temperature and $T_{\text {ref }}(K s)$, the local convective heat transfer coefficient can be computed from the ratio:

$h(x, y)=\left(e_{\mathrm{tot}}-e_{\mathrm{cond}}-e_{\mathrm{rad}}\right) / i_{\mathrm{temp}}$.

Moreover, a specific digital image restoration technique is developed to enhance the camera spatial resolution. The restoration technique uses a two-dimensional Wiener filter (Bougeard et al. 1994). The $e_{\text {tot }}$ image (Fig. 8a) shows the fin energy density lost during the experiment. High values are found at the fin leading edge because of the developing boundary layer and in front and around of the second tube row which are due to the horseshoe vortex presence. The $e_{\text {cond }}$ image (Fig. $8 \mathrm{~b}$ ) reveals high heat transfer by conduction around the tubes because of high spatial temperature variations due to the vortex structures. On the fin's remaining part, the conduction is quite small, isolated sharp variations being due to the presence of noise in infrared images. The $e_{\text {rad }}$ image (Fig. 8c) shows that radiation heat transfer is fairly low (temperatures are relatively low), representing only a few percent of the total energy exchange, and is predominant downstream of the tubes, where convection is low. The convective heat transfer coefficient distribution (Fig. 8e), computed according to (20), shows that: the first row of tubes has a very small horseshoe vortex effect; a heat transfer increase is found at the fin leading edge; the staggering disposition of tubes decreases the wake size behind their first row.
As an example of image restoration for the IR system modulation applied to the thin-film sensor (de Luca et al. 1990a, b), Fig. 9a shows the coarse footprints $\left(T\right.$ in $\left.{ }^{\circ} \mathrm{C}\right)$ of Goertler vortices on a solid $15^{\circ} \mathrm{RTV}^{\mathrm{TM}}$ elastomer ramp as recorded by the camera. The ramp is placed downstream of a delta wing in hypersonic flow at $M=8.15$ (with fluid moving from thermogram bottom upwards) and vortices are barely evident by the periodic horizontal variation of wall temperature. Even if the IR used interlaced camera (AGEMA 880LW) with extension rings had a limited spatial resolution $(140 \times 140$ pixels $)$, the restored image of Fig. 9b allows detecting the rather regular vortices structure which shows a mean pitch of about $2 \mathrm{~mm}$. Horizontal striping is due to camera interlacing and transient heating.

\section{Applications}

The applications of IR thermography include a much diversified thermo-fluid-dynamic phenomenology which spans from turbine cooling, including film cooling, to transition to turbulence and flow separation, micro systems, rotating bodies, jets, flow instability, two-phase and hypersonic flows. In Table 1 are indicated most of the contributions, appearing during the last two decades, to either measure wall convective heat fluxes or investigate surface flow field behavior, using IR thermography. These papers are organized according to various types of fluid flows they deal with, some of them appearing in more than one topic. Whenever possible, for each paper the adopted heat flux sensor and IR detector type are indicated. In the following, a few significant papers of some of these topics regarding complex fluid flows are presented and reviewed, with the aim as well of pointing out some relevant aspects.

\subsection{Transient natural convection}

The heated-thin-foil technique for analyzing transient natural convection on a vertical plate is described by Carlomagno and de Luca (1989). A vertical stainless steel foil (245 mm high, $960 \mathrm{~mm}$ wide, $40 \mu \mathrm{m}$ thick), coated on the viewed side with a thin layer of high emissivity $(\varepsilon=0.95)$ paint, is electrically step heated $\left(Q_{j}=0-80\right.$ to $\left.270 \mathrm{~W} / \mathrm{m}^{2}\right)$. By measuring the apparent temperatures on both foil sides, the uncoated side emissivity is evaluated. In fact, for the tested conditions, the radiative heat flux is of the same order of magnitude of the convective one and therefore, it must be definitely subtracted from the Joule heat input.

For a step Joule power input $0-130.5 \mathrm{~W} / \mathrm{m}^{2}$, the time evolution of the foil temperature vertical profile is shown in the pseudo-thermogram of Fig. 10 where abscissa is time 
Fig. 8 Plate fin and two-tube rows assembly (flow from left to right): a total energy

exchanged, $\mathbf{b}$ energy exchanged by conduction, $\mathbf{c}$ energy exchanged by radiation, d temperature integration and e computed convective heat transfer coefficient distribution. From Bougeard (2007)
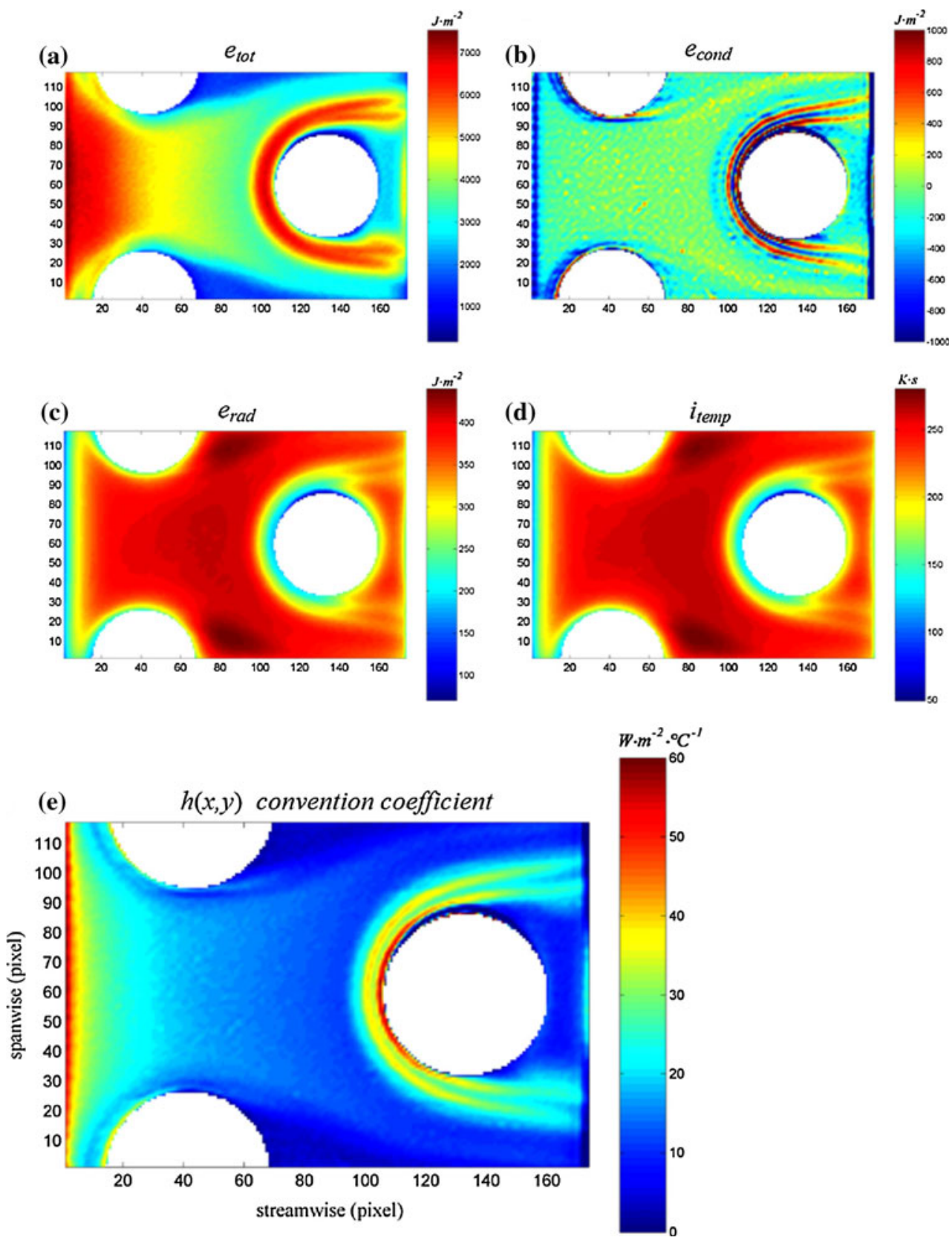

(for a total of $80 \mathrm{~s}$ ), ordinate is position along the foil central vertical segment $(0-245 \mathrm{~mm})$ and colors represent temperatures $\left(21-42^{\circ} \mathrm{C}\right)$. The thermogram's, left region, characterized by a sequence of colored vertical bands, points out an initial uniform time increasing of foil temperature which indicates a prevalent conductive heat transfer regime between foil and ambient air. Temperature evolution agrees well with theoretical calculation. The temperature variation, which later occurs in the vertical direction reveals the progressive onset of the natural convection flow. When steady state is reached, measured data agree within $\pm 5 \%$ with the theoretical prediction of Sparrow and Gregg (1956) for laminar flow.

\subsection{Film cooling}

High-pressure ratio and high temperature rise are required to improve the performance of modern gas turbines and impose strong challenges on combustor and initial turbine stages design. The combustor and the blades' leading edge are largely cooled by effusion (film) cooling that maintains adequate metal temperatures. This is achieved by injecting through a large number of holes cold air which produces a shielding film on the hot side surface. The performance of such a method is measured by the surface adiabatic film cooling effectiveness $\eta$, which in the most general case is defined as: 

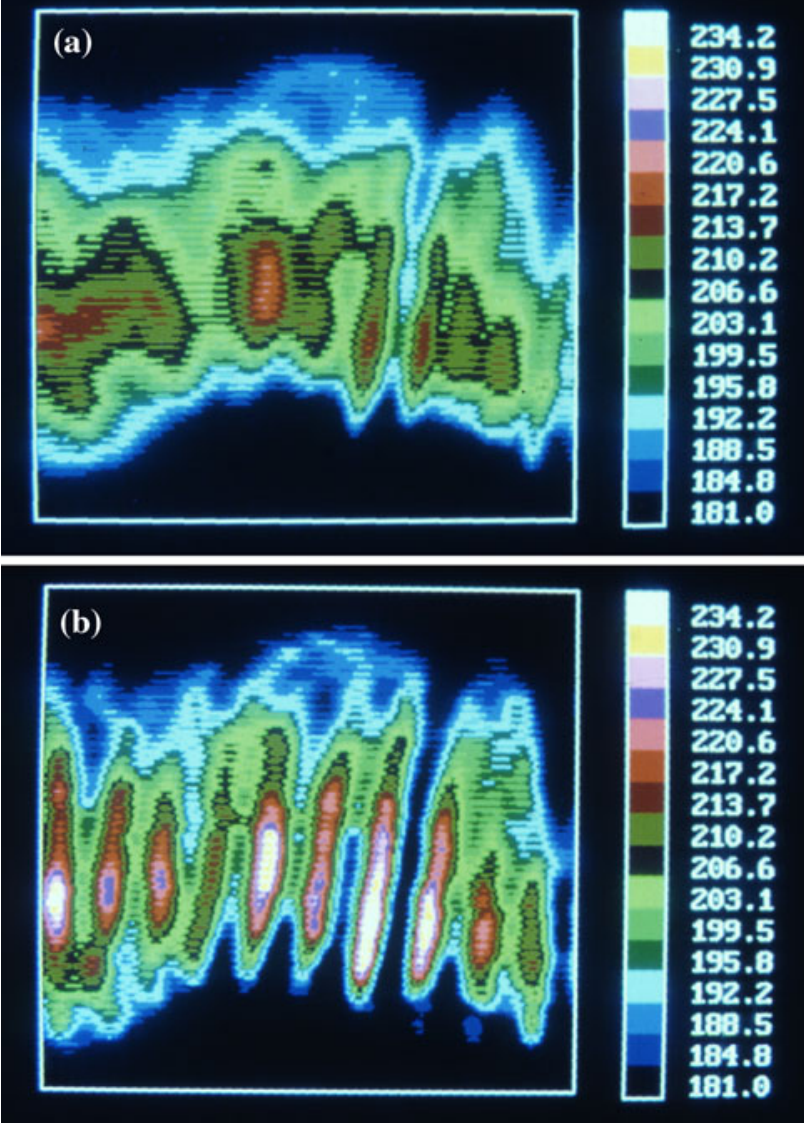

Fig. 9 Images of Goertler vortices on a $15^{\circ}$ ramp in hypersonic flow at $M=8.15$ : a coarse; $\mathbf{b}$ restored. From de Luca et al. (1990a)

$\eta=\left(T_{w}-T_{\mathrm{aw} \infty}\right) /\left(T_{o c}-T_{\mathrm{aw} \infty}\right)$

where $T_{\mathrm{w}}$ is the measured surface temperature, $T_{\mathrm{aw} \infty}$ is the free-stream adiabatic wall temperature, and $T_{o c}$ is the coolant stagnation temperature.

One of the first test rig to measure $\eta$ by means of IR thermography is presented by Leger et al. (1996). The test facility (Fig. 11) has a multi-hole cooling set up on a flat plate simulating the combustion chamber wall. The plate is subjected on one side to hot gases resulting from combustion (débit chaud) and on the other side to the cooling airflow (débit froid). The $\mathrm{CaF}_{2}$ infrared window (hublot IR) for the MWIR band is also visible. Calibration has to also take into account the presence of the IR window.

A recent study by Dhungel et al. (2009) deals with the film cooling performance of a flat plate with a row of simple holes and with the same holes, either complemented with two smaller symmetrical anti-vortex holes, which branch out from the main hole, or shaped at their exit. The proposed anti-vortex design requires only easily machinable round holes, unlike shaped holes, and is intended to counteract the detrimental vorticity associated with standard circular cross-section holes. Experiments are

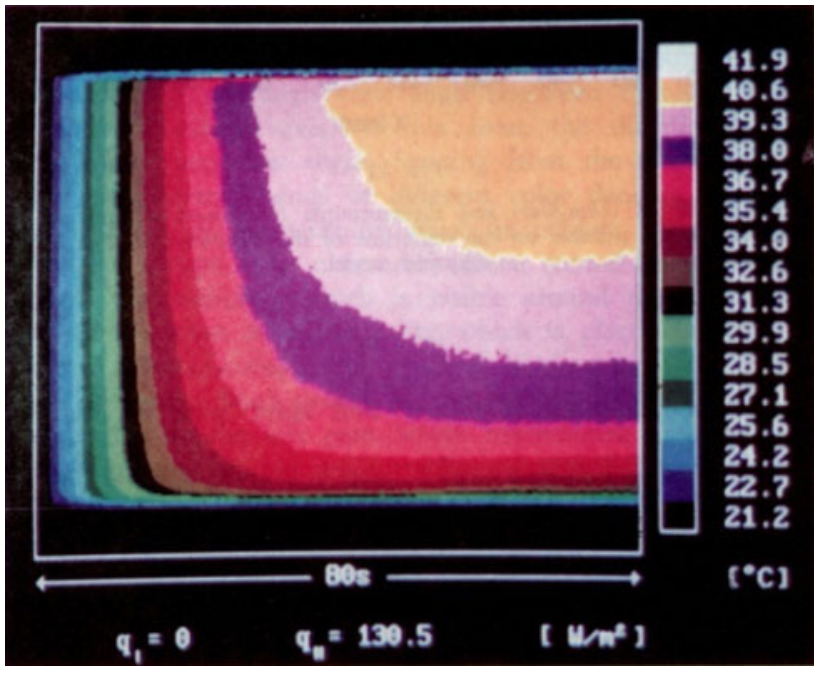

Fig. 10 Time evolution of temperature profile on a vertical plate under transient natural convection. From Carlomagno and de Luca (1991)

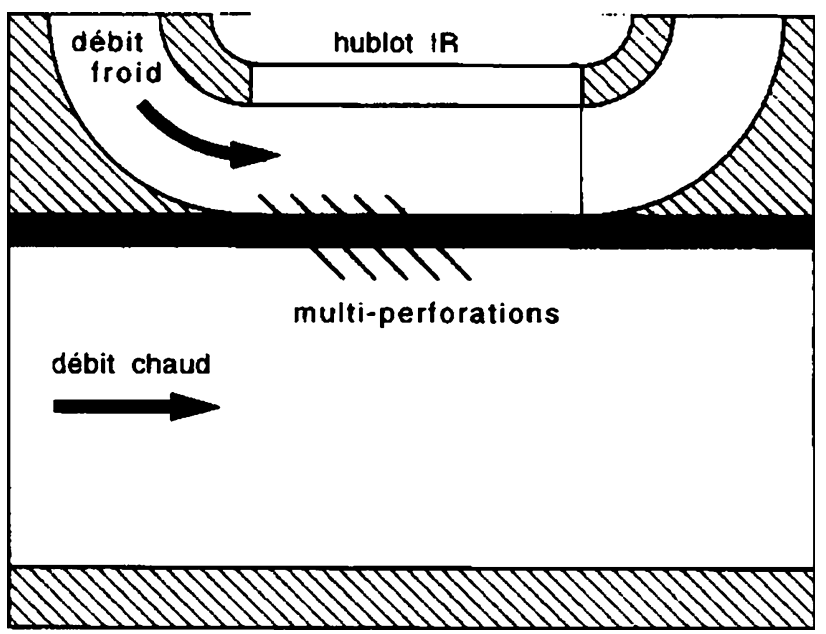

Fig. 11 LARA test bench by Leger et al. (1996)

performed at four different coolant-to-mainstream blowing ratios $B R$ from 0.5 to 2 by using the transient thin-film sensor.

The baseline test plate has six holes $(d=12.7 \mathrm{~mm})$ in each row, inclined at $30^{\circ}$ along the flow direction, hole spacing between adjacent holes being three-hole diameters. The anti-vortex holes are branching out of the main holes which have the same orientations and geometry as the baseline. Only the features of the anti-vortex holes are altered in six different geometries, some of their aspects being evident in the $\eta$ maps of Fig. 12. The shaped (diffuser exit) holes still have an inclination angle of $30^{\circ}$; the length of the cylindrical inlet portion twice the diameter of the hole and a compound angle of $15^{\circ}$. 
Fig. 12 Film cooling effectiveness distributions at different blowing ratios $B R$ : a 0.5 ; b 1.0 ; c 1.5 ; d 2.0. Flow from top to bottom. From Dhungel et al. (2009)

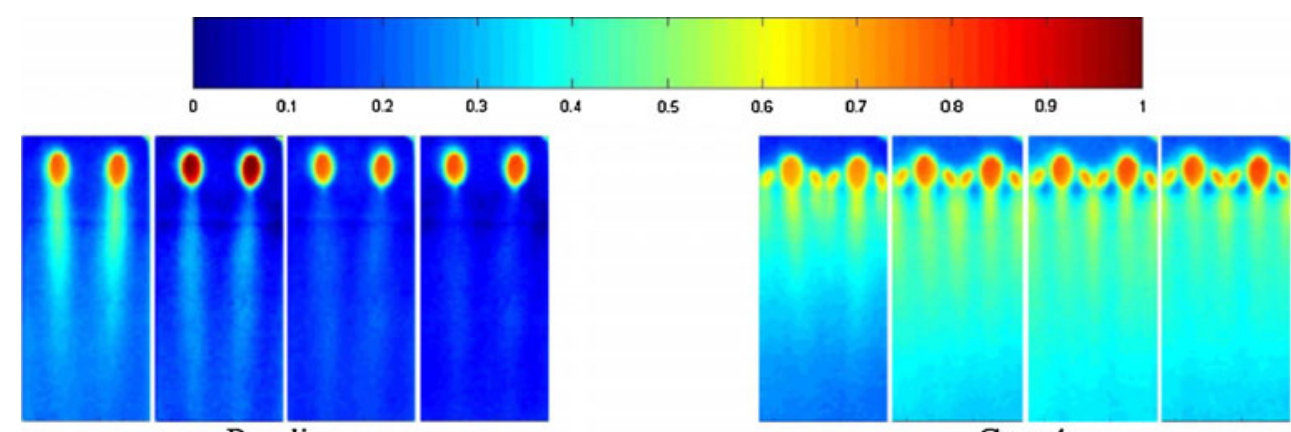

Baseline

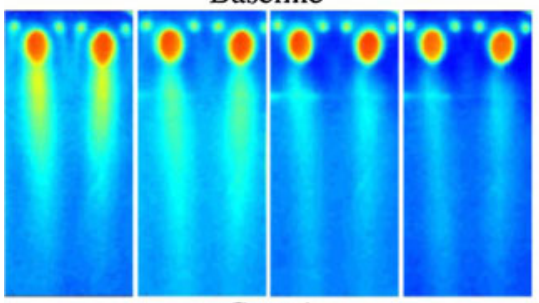

Case 1

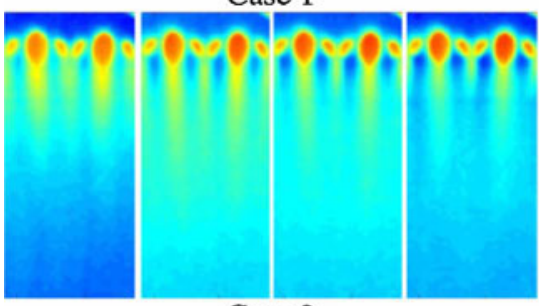

Case 2

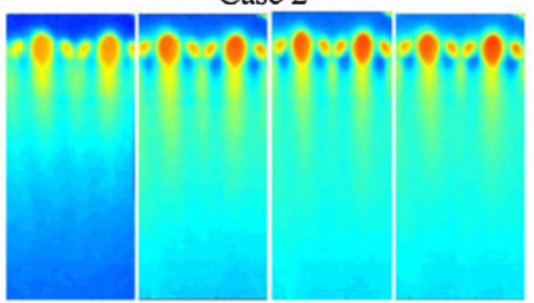

Case 3

(a)

(b)

(c)

(d)

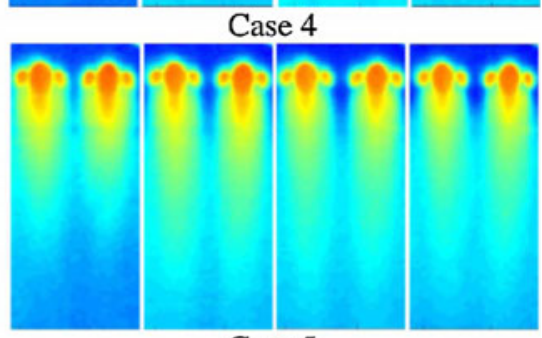

Case 5

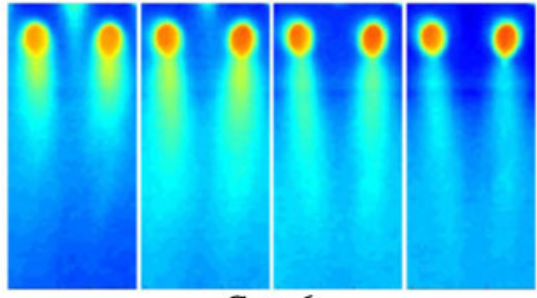

Case 6

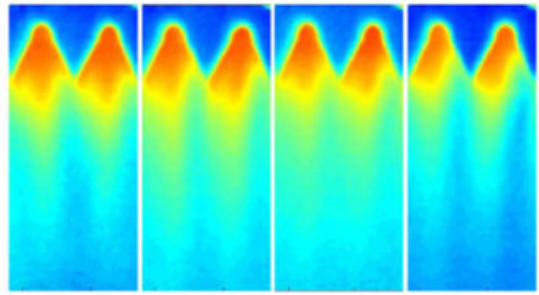

Shaped Holes

(a)

(c)

(d)

For the baseline case, the jet streaks are barely visible at high $B R$ where flow separation due to jet liftoff appears to occur, the highest effectiveness taking place when the jet has the lowest momentum flow rate, i.e. at $B R=0.5$. Case 1 has the anti-vortex holes with their exit located upstream of the main hole and 1/4 the diameter of this one. The trend is similar to the baseline case except for the overall higher $\eta$. The anti-vortex pair causes reduced flow through the main hole and also supplements the film coverage in the region between main holes.

For Case 2, the anti-vortex holes are 1/2 the diameter of the main holes and their entry is halfway into the main hole. The best film cooling effectiveness occurs for $\mathrm{BR}=1$. The two holes from the adjacent anti-vortex pairs interact and produce another jet streak similar to the main holes. Downstream, the various streaks coalesce producing a quasi-uniform spanwise effectiveness distribution. However, as the blowing ratio increases, the

effectiveness downstream gets worse. In Case 3, the antivortex holes have the same exit location and diameter as in case 2 but branch out from the base of the main holes so as to produce a more developed coolant flow. High film cooling effectiveness can be seen even for higher blowing ratios. The spanwise and downstream coverage increases with blowing ratio, the only drawback being that the region adjacent to the main holes remains uncooled.

Case 4 is similar to Case 3 except that the exit of antivortex holes is somehow more downstream than for Case 3. The result looks similar to that of Case 3, with a good attachment of the film even at higher blowing ratios. The additional length to the anti-vortex holes does not provide significant benefit even if there are some minor differences between Cases 3 and 4. Case 5 has the exit of anti-vortex holes at the same streamwise location of Case 3 but closer to the main holes. For all blowing ratios, the jets from the 
anti-vortex holes mix with those from the main holes, thus creating a thicker coolant streak footprint than in the other cases. However, the effectiveness distribution has not the lateral spread of Cases 3 and 4 and appears to peak at $\mathrm{BR}=1.5$ with similar levels at $\mathrm{BR}=2.0$.

The anti-vortex holes coming laterally outward from the base of the main holes, for Case 6 , have their exit at the same spanwise position of Cases 2-4 but much more upstream. At higher blowing ratios, the jets seem to clearly liftoff, but their presence causes a momentum decrease in the jet from the main hole, thus restricting the liftoff even at $\mathrm{BR}=1.5$. This causes a slightly better effectiveness than baseline but does not contribute to significant effects, such as those seen for Cases 2-4.

For shaped holes, the effectiveness distributions for blowing ratios of 1 and 1.5 indicate good lateral and downstream coverage. The larger exit area of these holes reduces the exit velocity of the coolant jet and hence the film remains adhered to the plate surface. However, for the highest blowing ratio, the coolant jet has greater momentum to liftoff, producing lower effectiveness than for $\mathrm{BR}=1.5$. At high blowing ratios, a classical separation, resulting in skewed effectiveness profiles inside the diffuser, exists especially on the right side.

\subsection{Impinging jets}

Impinging jets receive great attention from both industrial and academic points of view. Industrial interest is justified by the many applications such as to dissipate heat in electronic circuits, achieve high heat transfer coefficients at the leading edge of turbine blades, dry textiles, temper glass and heat zones which are critical for ice formation over aircrafts. Research attention derives from the complex fluid flow occurring in impinging jets.

With regard to IR measurement for a round impinging jet, the works by Meola et al. (1995, 1996, 2004), who report measurements of adiabatic wall temperature $T_{\text {aw }}$ and of heat transfer coefficients performed with the heatedthin-foil sensor, must be mentioned. The sensor (impingement plate) consists of a thin constantan foil (200 mm wide, $470 \mathrm{~mm}$ long, and $50 \mu \mathrm{m}$ thick), Joule heated and held flat by a stiffening fixture. Truncated cone nozzles are adopted with several exit diameters $d$ and nozzle-to-plate distances $z$. The jet Mach number $M$ ranges from 0.04 to 0.91 and the Reynolds number $R e=V d / v$ from 10,000 to 173,000 , both based on the nozzle exit velocity $V$. Total jet temperature is kept equal to the ambient one.

Meola et al. (1995), through measurements of adiabatic wall temperature $T_{\text {aw }}$ (not heated foil), observe instability developing for high $M$ values, such phenomena also depending strongly on the impingement distance. For $z / d<6$, as $M$ increases, the vortex ring, which is located in the shear layer at $r \approx 1.2 d$ ( $r$ is the radial coordinate), strengthens up and breaks (Widnall instability) entailing entrainment of warmer ambient air and giving rise to the formation of structures. Snapshots of the $T_{\mathrm{aw}}$ map $(d=5 \mathrm{~mm}, z / d=4)$ at different Mach number values are shown in Fig. 13, where several thermograms are reported. For $M=0.3$ (Fig. 13a), the minimum $T_{\mathrm{aw}}$ at $r \approx 1.2 d$ has the shape of a completely developed and stable annulus. This annulus, which is the location of the vortex ring, for $M=0.4$ is transformed into an unstable semicircle. As $M$ increases, the vortex ring reinforces and, for $M=0.71$ (Fig. 13d), breaks up in the impact with the plate, entraining warmer ambient air and giving rise to secondary minima at about 0.9 and $2.2 d$, with a maximum between them at about $1.6 d$. As $M$ further increases, such structures become stronger and reach their highest magnitude for $M=0.85$ (Fig. 13e). As $M$ further increases, the structures break up into numerous smaller structures, which tend to coalesce giving rise to a transient alternate circumferential movement.

The recovery factor is defined as $r_{f}=\left(T_{\mathrm{aw}}-T_{j}\right) /$ $\left(T_{o j}-T_{j}\right.$ ), where $T_{o j}$ and $T_{j}$ are the initial jet total and static temperatures, respectively. Carlomagno and de Luca (1989) report tests performed with $T_{o j}$ equal to ambient temperature and Fig. 14 shows the relief map of the recovery factor $r_{f}$, corresponding to $d=10 \mathrm{~mm}, z / d=8$, $R e=130,000$ and $M=0.52$. The recovery factor has a peak value on the jet axis and a local minimum in the annular region at $r / d \approx 1$, which is attributed to the vortex ring in the shear layer surrounding the jet. A local $r_{f}$ maximum is located at $r / d \approx 1.8$ which is followed by another minimum at $r / d \approx 3.8$. For further $r / d$ increasing, the recovery factor attains a unity value.

The relief map of the Nusselt number, for $d=10 \mathrm{~mm}$, $M=0.11, R e=28,000$ and a much lower distance $z / d=2$, is represented in Fig. 15. When the jet flow impinges on a wall relatively close to the nozzle exit, moving along the radial direction two local minima and two local maxima values are found. The minima are located one at the jet axis and the other one at $r / d \approx 1.5$ (practically where also the minimum of $r_{f}$ is placed). Instead, the inner maximum is positioned at $r / d \approx 0.5$ and the outer one at $r / d \approx 2.5$. This behavior confirms results documented in the literature.

An example of multi-jet impingement is described by Fénot et al. (2008), who report experimental measurements of heat transfer due to a row of hot air jets impinging on a concave semi-cylindrical surface performed by using a printed circuit board to implement the heated-thin-foil technique. They state that the effects of tangential conduction through the board are small. The influence of high relative curvature $(d / D=0.1-0.2)$ is investigated by 
Fig. 13 Adiabatic wall temperature for $d=5 \mathrm{~mm}$, $z / d=4$ and increasing Mach number; a $M=0.3 ; \mathbf{b} 0.4$; c 0.67 ; d 0.71 ; e $0.85 ;$ f 0.91 . From Meola et al. (1995)
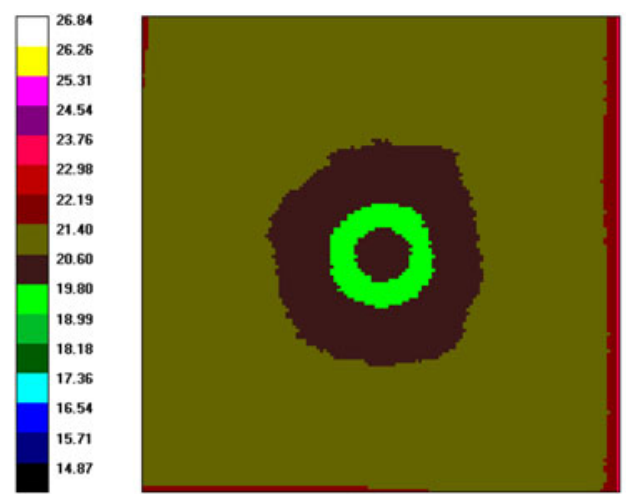

(a)
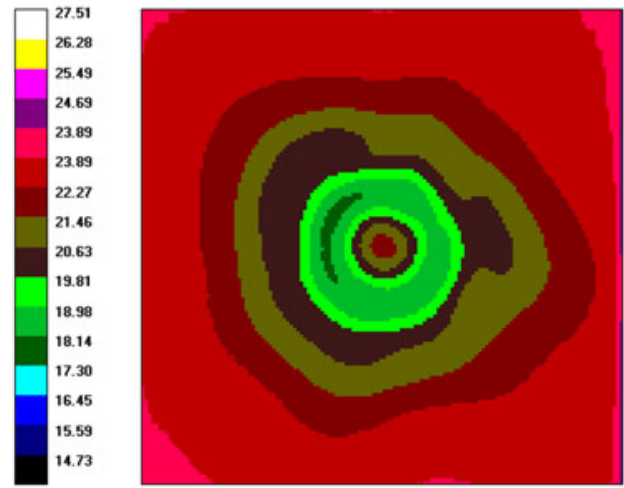

(c)
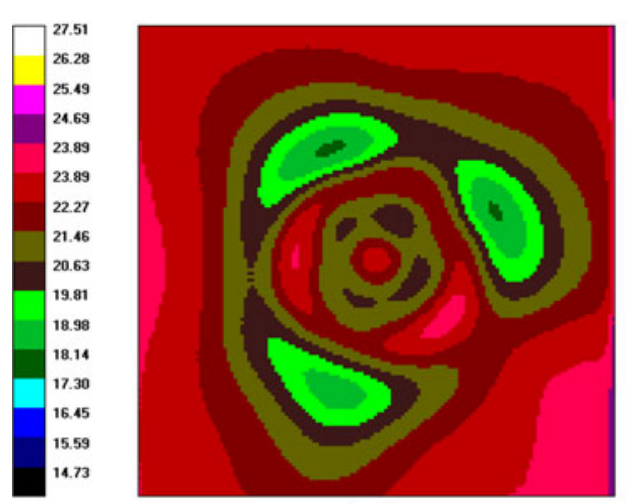

(e)

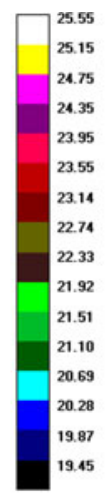

19.45

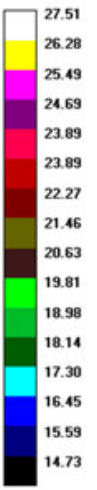

27.51
2.28
2.49
2.69
23.89
23.89
2.27
21.46
20.63
9.81
18.98
18.14
17.30
16.45
15.59
14.73

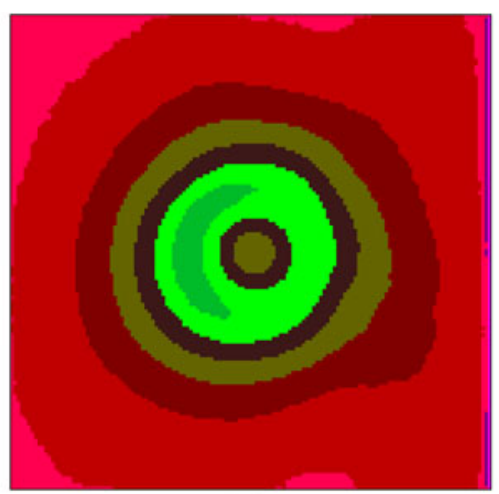

(b)

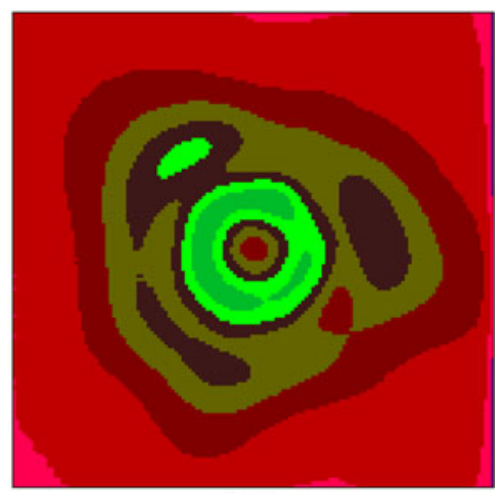

(d)
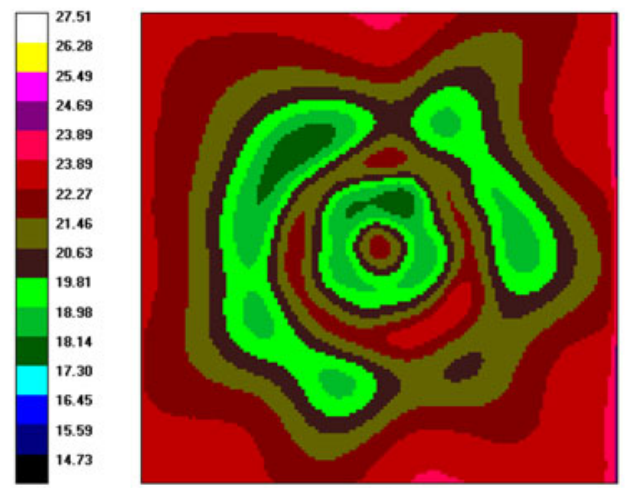

(f) changing jet tube diameter $d$, the impinging surface diameter $D$ remaining constant. Reynolds number based on $d$ and jet exit velocity, injection temperature, jet-to-jet spacing $P$ and impingement distance $z$ are also made to vary. Nusselt number is based on jet tube diameter $d$.

Typical effectiveness defined as $\eta=\left(T_{\mathrm{aw}}-T_{\mathrm{amb}}\right) /$ $\left(T_{j}-T_{\mathrm{amb}}\right)$, where $T_{\mathrm{amb}}$ and $T_{j}$ are ambient and jet temperature, respectively, together with Nusselt number $\mathrm{Nu}$ maps are presented in Fig. 16. The small circular zones without results (mainly visible in the (map) are zones covered with reflective adhesive used as spatial references. Spanwise periodicity and symmetry look satisfactory. The curvature produces a confinement of the jet flow that gives high $\eta$ values at the impinging point so adiabatic wall temperature $T_{\text {aw }}$ is nearly equal to injection temperature.

The Nusselt number map around impinging points has an eight-form and, going along the $s$ direction, $\mathrm{Nu}$ shows a local minimum at the impinging point, then attains a maximum at $s / d=0.4$ and afterward decreases. The maximum location roughly corresponds to the half-width of the jet potential core. Going along the $Y$ direction, at the impinging point $N u$ has a maximum, then weakly decreases and finally shows a small local maximum halfway between two adjacent jets. 


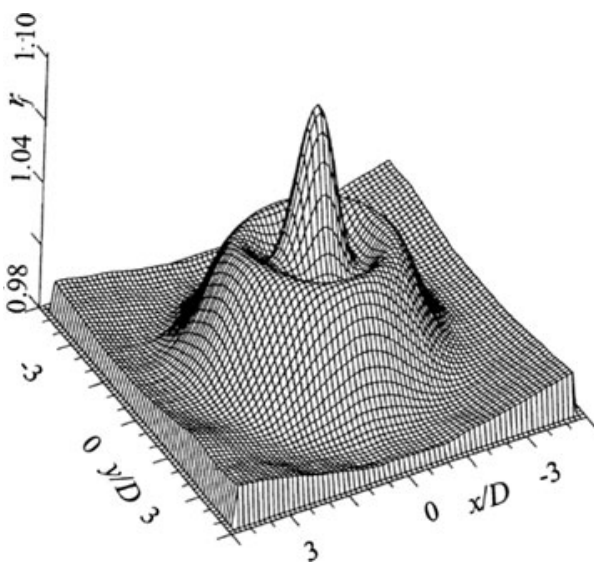

Fig. 14 Recovery factor $r_{\mathrm{f}}$ relief map of a single jet impinging on a flat plate for $d=10 \mathrm{~mm} ; z / d=8 ; M=0.52 ; R e=130,000$. From Carlomagno and de Luca (1989)

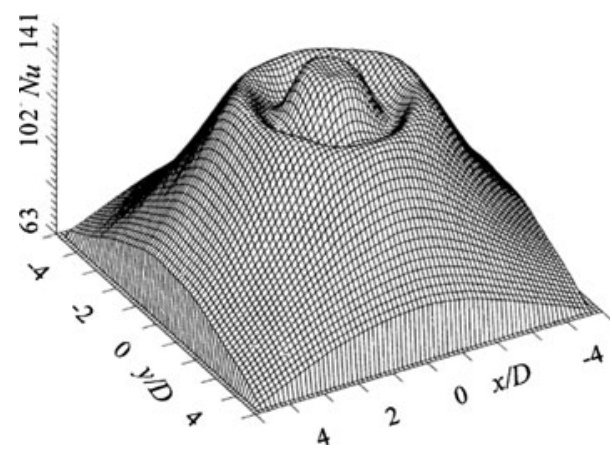

Fig. 15 Nusselt number relief map of a single jet impinging on a flat plate for $d=10 \mathrm{~mm} ; z / d=2 ; \quad M=0.11 ; R e=28,000$. From Carlomagno and de Luca (1989)

Another example of multi-jet impinging is presented by Roger (2007) who describes a periodic transient technique for high-resolution heat transfer measurement. In contrast to other measurement techniques, this one requires neither homogenous heating nor quantitative measurement of surface or fluid temperatures. The heat transfer coefficient is determined by periodically heating the sensor and evaluating the phase shift between the heat flux penetrating the sensor surface and the resulting temperature response. The technique is applied to a hemispherical solar receiver window, with a 5-mm thick fused silica sensor, a radius of $0.31 \mathrm{~m}$ and multiple air jets cooling the concave surface.

The convective heat transfer coefficient map with a sixnozzle $(d=8 \mathrm{~mm})$ symmetric window-cooling configuration is shown in Fig. 17. The bottom window edge is assigned a zero $h$ value because no measurements are possible there. Since IR camera has a limited field of view and measurements are periodic, the map of Fig. 17 is composed of six identical $60^{\circ}$ slices. High heat transfer coefficients are observed where jets impinge with a kind of

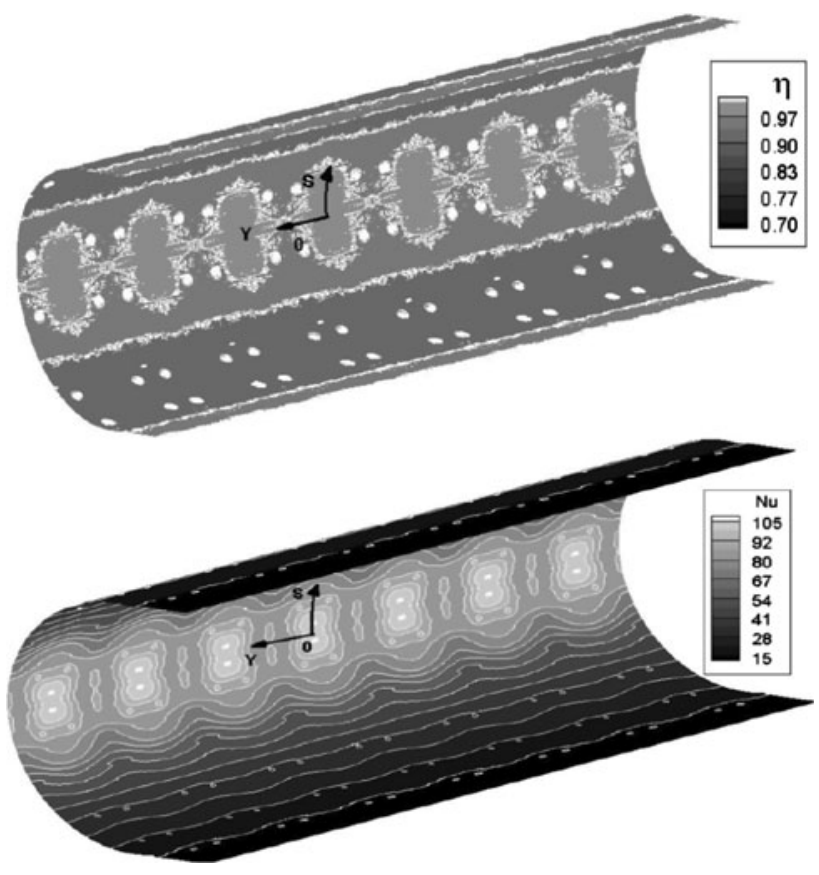

Fig. 16 Effectiveness $\eta$ and Nusselt number maps $(R e=23,000$, $\left.z / d=2, P / d=4, d / D=0.1, T_{j}-T_{\mathrm{amb}}=38^{\circ} \mathrm{C}\right)$. From Fénot et al. (2008)

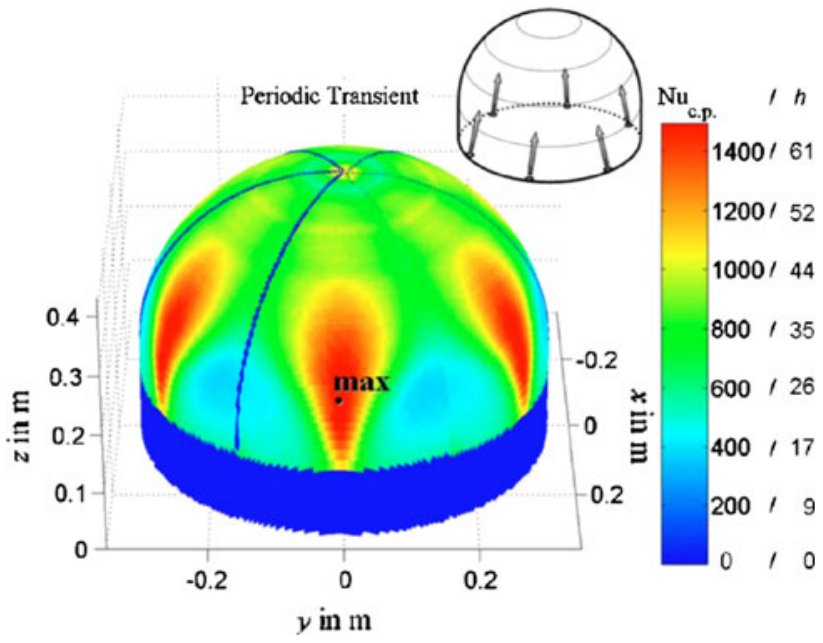

Fig. 17 Heat transfer map on the window surface for symmetric airjet cooling with six steady round-type nozzles; $d=8 \mathrm{~mm}$; $V=47 \mathrm{~m} / \mathrm{s} ; R e=23,200 ; \mathrm{h}$ in $\mathrm{W} /\left(\mathrm{m}^{2} \mathrm{~K}\right)$. From Roger $(2007)$

Coanda effect on the wall, while, between the jets, the heat transfer is much lower. At the window top, a stagnation point causes only fair heat transfer.

\subsection{Airfoils Transition/Separation}

The knowledge of the temperature distribution over an aerodynamic body is of great interest because of the contained information which can be related to heat transfer and 
skin friction at the wall through Reynolds analogy and to detect transition, separation and reattachment zones.

de Luca et al. (1990b) study the flow field over a Göttingen 797 airfoil (with a $180 \mathrm{~mm}$ chord) at several angles of attack. The wing model is made of a glass-epoxy skin ( $\approx 3 \mathrm{~mm}$ thick) over polyurethane foam. In order to perform measurements by means of the heated-thin-foil sensor, the wing leeside is coated with a Joule heated thin $(30 \mu \mathrm{m})$ stainless-steel foil, $155 \mathrm{~mm}$ wide, which starts $13 \mathrm{~mm}$ away from the leading edge and is coated with a very thin layer of thermally black paint $(\varepsilon=0.95)$. Tests are carried out in a Göttingen type closed-return tunnel with circular open test section $1.1 \mathrm{~m}$ in diameter. Results are reduced in non-dimensional form in terms of the Stanton number $S t$ defined in the conventional way.

The chordwise $S t$ profiles at leeside for chord based Reynolds number $R e=259,000$ and different angles of attack $\alpha$ (in the range $-12^{\circ}$ to $20^{\circ}$ ) are shown in Fig. 18 . The beginning of the plateau may be assumed to coincide with the point of laminar separation and its end with the beginning of transition in the separated shear layer. As $\alpha$ increases the minimum $S t$ value moves upstream following the movement of the laminar separation point deriving from the adverse pressure gradient on the airfoil leeside. At the highest $\alpha$ value, the plateau disappears from the measured zone. Data are slightly affected by tangential conduction in the model wall and by the modulation transfer function of the IR scanner that, as stated by the authors, has a limited spatial resolution. They were both not corrected at the time.

Ricci and Montelpare (2005) carry out similar tests on the laminar bubble occurrence by also using the heatedthin-foil sensor. The laminar bubble is a local boundary layer separation phenomenon that mainly exists at low Reynolds numbers. Three characteristic points of the bubble are searched for: the laminar separation point, the transition point and the turbulent reattachment point. The laminar bubble behavior is tested for an RR3823HL airfoil at

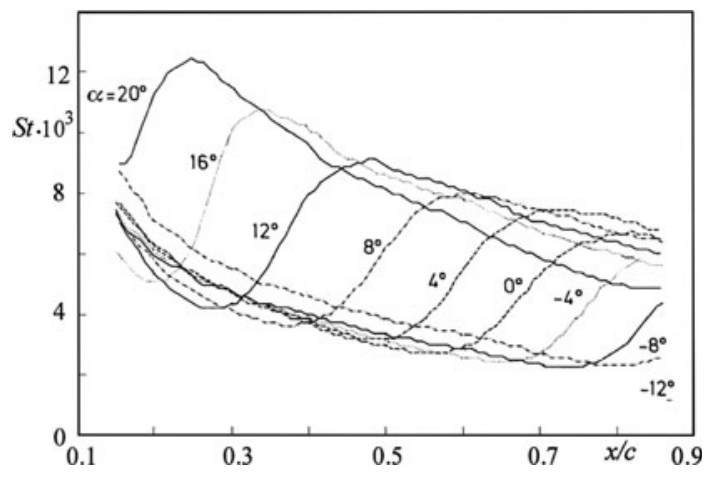

Fig. 18 Stanton number chordwise distribution over leeside of Gottingen 797 airfoil at several angles of attack. Flow from left to right. From de Luca et al. (1990b)
Reynolds number based on the airfoil chord equal to 200,000 .

In order to individuate the searched points in a simple and objective way, results are presented in terms of a modified Stanton number. In fact, to enhance with greater detail the Stanton number variations along the chord, instead of computing $S t$ with the free stream speed $V_{\infty}$, the authors calculate its values with the inviscid speed $V_{i}(s)$, i.e., the speed the flow attains outside the boundary layer along the curvilinear chordwise abscissa $s . V_{i}(s)$ is evaluated with the inviscid solution of Xfoil code, the choice deriving from the fact that the computed speed is independent of the code used. Once measured the St profile along the chord (see Fig. 19), the laminar separation point is localized as the flex point that comes before the absolute minimum. In fact, since the laminar separation represents a fluid dynamic singularity, this also reflects in a singularity for the thermal heat exchange. The boundary layer transition point is localized at the absolute minimum $S t$ value because it corresponds to the point of maximum bubble height, where the convective heat transfer coefficient reaches its lowest value. The turbulent reattachment point location is similarly individuated by the maximum heat transfer coefficient because the flow behaves there like an impinging jet. The upper part of Fig. 19 shows the recorded thermogram of the airfoil (blue: low temperature, high heat transfer; red: high temperature, low heat transfer).

Zuccher and Saric (2008) perform a qualitative study on the boundary-layer transition in supersonic flows by testing several models (having an initial temperature difference with respect to the oncoming flow) of swept wings in a blow-down facility at Mach number 2.4. They observe effects of wing sweep and other parameters such as angle of attack, leading edge contour and presence/absence of surface roughness. The IR technique is revealed to be very effective for flows sensitive to surface roughness, such as

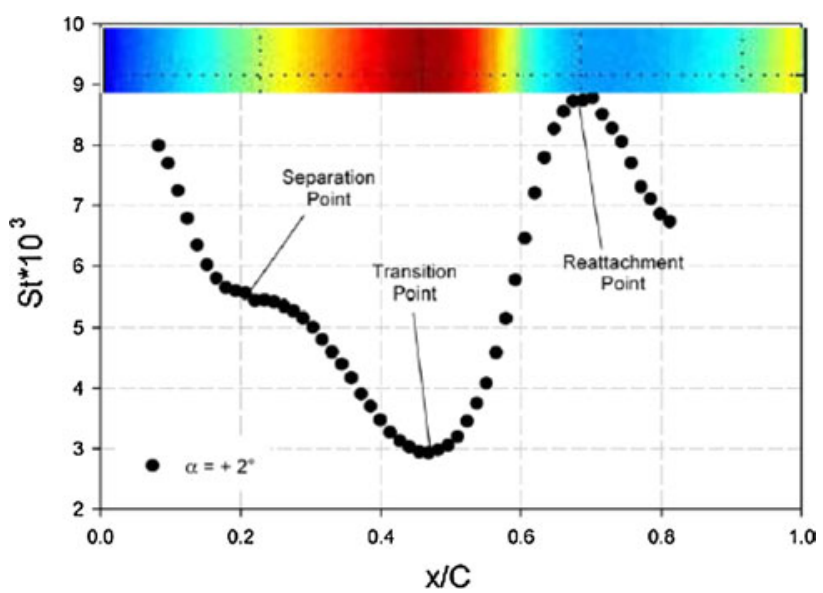

Fig. 19 Stanton number vs. airfoil chord. Flow from left to right. From Ricci and Montelpare (2005) 
transitional boundary layers, since it does not require interaction with the model or the flow and because it can be applied also by researchers not very familiar with IR thermography and heat transfer.

Figure 20 shows a typical IR image acquired for a swept angle equal to $20^{\circ}, \alpha=0^{\circ}, M=2.4$ and $R e_{\mathrm{u}}=31.2 \times 10^{6} / \mathrm{m}$. Since the external flow is at a lower temperature than the model and the (downstream) laminar boundary layer has a low heat transfer coefficient, the laminar region appears warmer (about $12^{\circ} \mathrm{C}$ ) than the turbulent one (about $8^{\circ} \mathrm{C}$ ). The transition front is visible at about $50 \mathrm{~mm}$ from the leading edge, i.e. $\sim 8.5 \%$ of the chord but is not uniform because of the presence of turbulent wedges, originating from very small disturbances on the leading edge, which move when the model is polished. A reflected wave is also visible in the upper-right portion of the image, together with an anticipated transition region. The authors claim that the leading edge is much colder (even if a narrow warmer strip appears at its periphery) than the rest of the model not because of turbulent flow, but because of its very low thermal inertia which lets it reach the external lower temperature much faster than the rest of the model. They seem to overlook the fact that, in laminar flow, the Stanton number tends to peak up moving toward the wing leading edge (as shown e.g. in Figs. 18 and 19), nor do they mention the adiabatic wall temperature variation between laminar and turbulent flow, which, for high Mach number such as the one they are testing at, is very relevant (see Sect. 4).

\section{$6.5180^{\circ}$ turn channels and ribs}

As already affirmed in Sect. 6.2, to improve the performance of modern gas turbines, high temperatures are

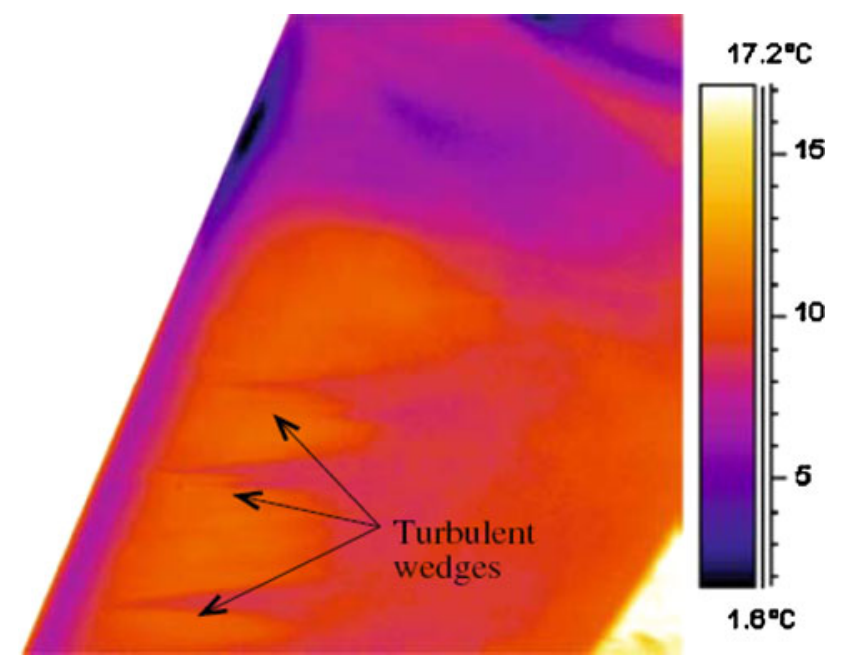

Fig. $2020^{\circ}$ Swept-wing phenolic model $\left(M=2.4 ; R e_{\mathrm{u}}=31.2 \times\right.$ $10^{6} / \mathrm{m} ; \alpha=0^{\circ}$ ). Flow from left to right. From Zuccher and Saric (2007) required. Often, to cool blade surface, air from the compressor goes through the hub section into the blade interior and, after flowing through serpentine passages, is discharged into the external gas. These passages are mostly made of several adjacent straight ducts, spanwise aligned along the blade and connected by $180^{\circ}$ turns. The turns cause flow separation and reattachment and induce secondary flows so that the convective heat transfer coefficient exhibits high local variations with consequent increased wall thermal stresses.

With the heated-thin-foil sensor, Astarita and Cardone (2000) obtain local heat transfer measurements on a $180^{\circ}$ sharp turn of a square smooth channel. Reynolds number $R e$ is based on hydraulic diameter $(D=80 \mathrm{~mm})$. Heat transfer coefficients are presented in terms of the normalized local Nusselt number $N u / N u^{*}$, where $N u^{*}$ is the Nusselt number as predicted by Dittus and Böelter correlation for channel flows.

The $N u / N u^{*}$ distribution is presented in Fig. 21 for Reynolds number $R e=30,000$. Data is corrected for radiation, tangential conduction and natural convection at the sensor backside, which is the viewed surface. Flow enters the duct from the left channel (inlet) and exits from the right one (outlet). At the inlet channel, the flow is fully developed so the heat transfer coefficient is about that predicted by Dittus and Böelter correlation. The flow field in the turn region, and downstream of it, is highly three-

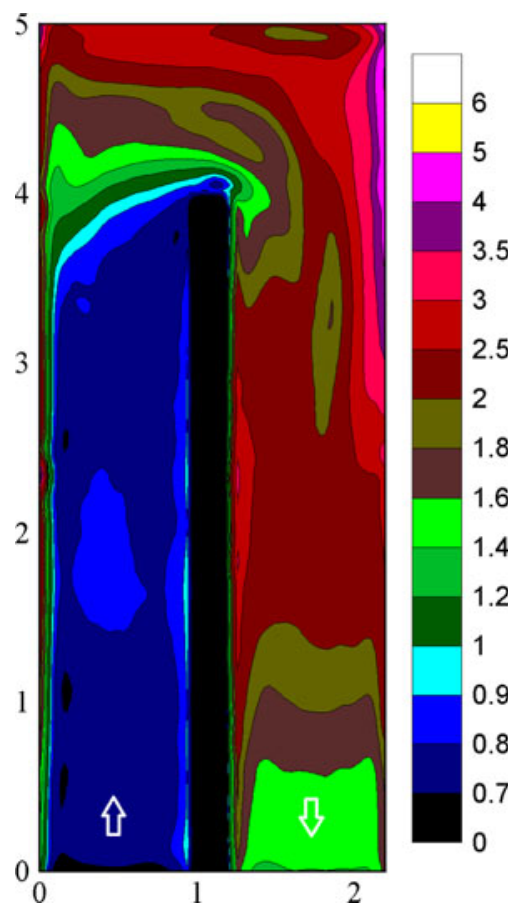

Fig. 21 Normalized Nusselt number $\mathrm{Nu} / \mathrm{Nu} *$ distribution for smooth channel: $\mathrm{AR}=1, \operatorname{Re}=30,000$, symmetric heating. Flow enters from left and exits to right. From Astarita and Cardone (2000) 
dimensional and the normalized $N u$ map shows zones with a relatively low heat transfer coefficient, corresponding to recirculation bubbles, as well as zones with high heat transfer coefficient, which are due to flow reattachment and/or an increase in the flow mean turbulent level. A first recirculation bubble is located just before the first outer corner and attached to the external wall. Two other regions with relatively low heat transfer coefficient may be noticed, the first nearby the partition wall tip and the second by the end wall by the outlet channel axis. Three high heat transfer zones are also observed: the first placed near the end wall; the second downward of the second outer corner and extending for about 3 diameters; the third about 2 diameters after the second inner corner and attached to the partition wall. They are due to the jet effect of the flow through the bend.

Rib turbulators attached to the heat exchanging walls enhance convective heat transfer and completely modify the channel flow field as well as the local heat transfer coefficient distribution.

For the channel described earlier with angled rib turbulators, Astarita et al. (2002a) and Astarita and Cardone (2003) present detailed quantitative maps of the heat transfer distribution again obtained with the heated-thinfoil sensor. The aluminum ribs have a square side ( $e=8 \mathrm{~mm}$ ), are placed on the two opposite walls, at an angle of $60^{\circ}$ with respect to the duct axis and at a pitch $P=80 \mathrm{~mm}$. Data are corrected for radiation, tangential conduction and natural convection at the sensor back side.

The two-dimensional map of the local normalized Nusselt number $N u / N u^{*}$ is shown in Fig. 22 for $R e=30,000$. Ribs are clearly visible due to the higher heat transfer rate occurring over them. The low $\mathrm{Nu}$ value spots on the last rib of the inlet duct and on the first rib of the outlet duct, near the partition wall, are due to a partial detachment of the ribs glued to the wall, owing to high local thermal stresses difficult to avoid. The authors believe that this has little influence on the local nearby measurements, but it has to be highlighted that it is easily revealed by IR thermography. Before the turn, the thermal pattern in the inlet duct appears to be recurring (a kind of thermally fully developed flow) except for some edge effects at the duct entrance and exit, due to the quite strong tangential conduction owing to dissimilar nearby zones there. The map also shows that the sharp turn presence already induces a slight modification of the shape of the contour lines before the inlet duct last rib.

The rib angle causes the formation of secondary flows under the form of two counter-rotating vortices in the channel cross-section. In the inlet duct, the main flow nearby both the bottom and top walls, entrapped by the ribs, is accelerated toward the sidewall. The secondary flows after licking this wall merge and go back, via the duct

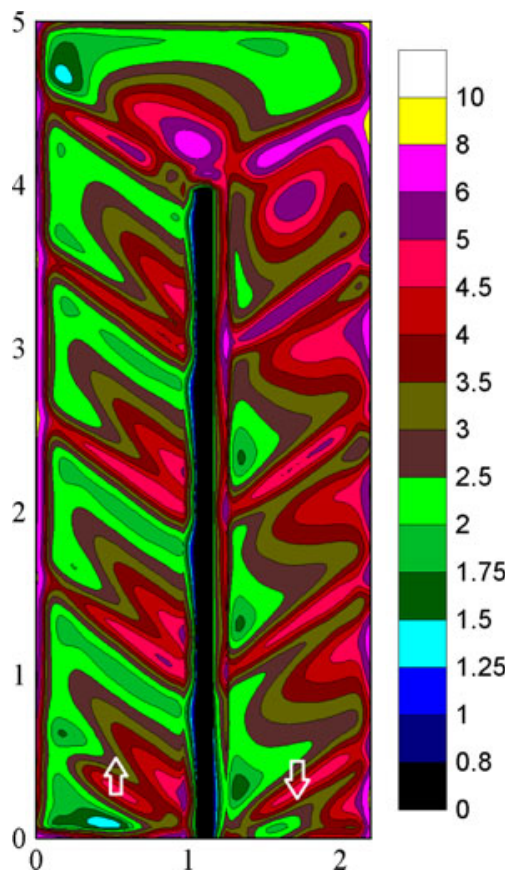

Fig. 22 Nusselt number $N u / N u^{*}$ distribution for $R e=30,000$, rib angle $60^{\circ}, P / e=10$. Flow enters from left and exits to right. From Astarita et al. (2002a)

center plane, to the partition wall so as to practically generate a jet that impinges onto the latter wall. This explains the ribwise $\mathrm{Nu}$ asymmetry. In fact, the jet presence tends to increase the convective heat transfer coefficient near the partition wall with respect to that near the sidewall. The map shows also that, toward the channel exit, the secondary flows which are reversed enhance the convective heat transfer coefficient near the side wall with respect to that near the partition wall.

In the inlet duct, the reattachment downstream of the ribs can be identified as the locus of the normalized Nusselt number local maxima when moving streamwise. The reattachment distance increase going toward the sidewall is due to the interaction of the main flow with the secondary one. The separation zone after each rib is strongly influenced by the secondary flow impingement toward the partition wall. Instead, the separation zone ahead of each rib remains well visible and it is not much influenced by the secondary flow, its width remaining practically constant along the ribs.

The heated-thin-foil sensor to determine local heat transfer distributions is used also by Gupta et al. (2008) in a double wall ribbed square channel for several configurations and by varying the pitch to rib height ratio $(P / e)$ and the rib height to hydraulic diameter ratio $(e / D)$. Reynolds number based on $D$ ranges from 10,000 to 30,000 . Results are again presented in terms of normalized Nusselt number $\mathrm{Nu} / \mathrm{Nu}$ *. 
Fig. $2360^{\circ} V$-broken ribs $e / D=0.0625, P / e=10$ : a Local heat transfer profiles; b Map of local heat transfer. Flow from left to right. From Gupta et al. (2008)

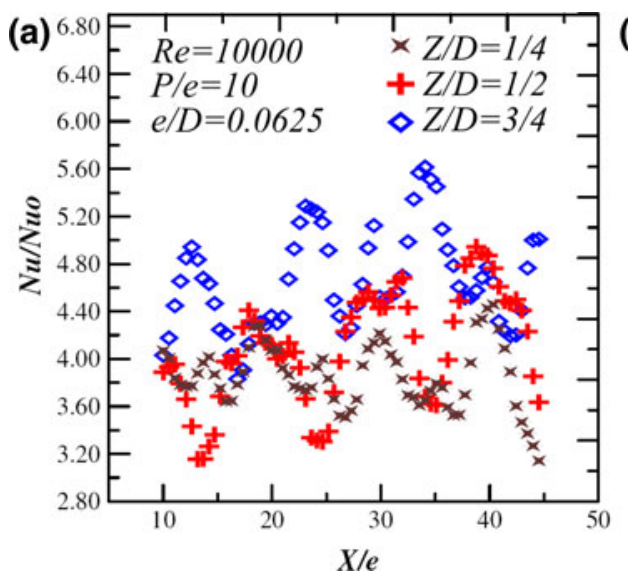

For $P / e=10$, local profiles of $N u / N u^{*}$ for the $60^{\circ}$ V-broken rib configuration in the streamwise direction are presented in Fig. 23a. The corresponding $\mathrm{Nu} / \mathrm{Nu}$ * maps are shown in Fig. 23b, where some asymmetries appear. Spanwise variations are high indicating that the presence of ribs distorts a lot the thermo-fluid-dynamic field. Lobes of heat transfer enhancements along the streamwise direction are ascribed to the secondary flow developed in the channel due to the rib shape. The generated vortices strongly mix the flow and hence heat transfer rates from the surface are enhanced.

Blades rotation changes the channel fluid dynamic behavior because there is an interaction between the Coriolis forces, pressure gradients causing main flow and centrifugal forces. For a radially outward flow, the Coriolis force produces a secondary flow (i.e., a symmetric pair of secondary vortices) in the plane normal to main flow direction which pushes the particles in the channel center toward the trailing (that follows) wall, then the flow continues along the sidewalls and finally gets to the leading (that goes ahead) wall. When the flow is reversed, i.e. for a radially inward flow, one has to change the role played by the leading wall with that of the trailing one and vice versa.

One of the first attempts to measure convective heat transfer coefficients $h$ in a rotating air channel with IR thermography is performed by Cardone et al. (1998). The apparatus concept is a direct consequence of the used heated-thin-foil sensor. Since the foil back surface (to be viewed by the IR camera) cannot be thermally insulated, to avoid here high thermal losses by forced convection, the channel rotates in a vacuum tank (below $100 \mathrm{~Pa}$ ).

Also Gallo et al. (2007) measure $h$ maps nearby a $180^{\circ}$ sharp turn of a rotating channel with the heated-thin-foil sensor. Their experimental apparatus (Fig. 24) consists of a Plexiglas two-pass water channel with a sharp $180^{\circ}$ turn, mounted on a revolving platform whose rotational speed can be continuously varied in the range $0-60 \mathrm{rpm}$. The channel has a square cross-section $60 \mathrm{~mm}$ on a side, its length of $1,200 \mathrm{~mm}$ ahead of the $180^{\circ}$ turn ensuring a hydro-dynamically fully developed flow before the turn. The central partition wall dividing two adjacent ducts is $12 \mathrm{~mm}$ thick. Water from a tank is pumped through an orifice meter, a rotating hydraulic coupling and, after flowing in the test channel, is discharged back into the tank. A magnetic pick-up synchronizes IR image acquisition. The classical heated-thin-foil reducing technique is modified because water presence makes the Biot number not sufficiently low. The apparatus is capable of reproducing both Reynolds numbers and rotation numbers $R o=\varpi D / V$ (where $\varpi$ is the rotational speed of the channel) values typical of turbine blades.

In Fig. 25, the segment-by-segment average $\mathrm{Nu} / \mathrm{Nu}$ * profiles at the leading and the trailing channel walls, for $R e=20,000$ and rotation number $R o$ in the range $0.0-0.3$, are reported. The length of a segment is half channel side. In the inlet duct, the flow results fully developed for both walls being $\mathrm{Nu} / \mathrm{Nu}$ * practically constant in the first four segments.

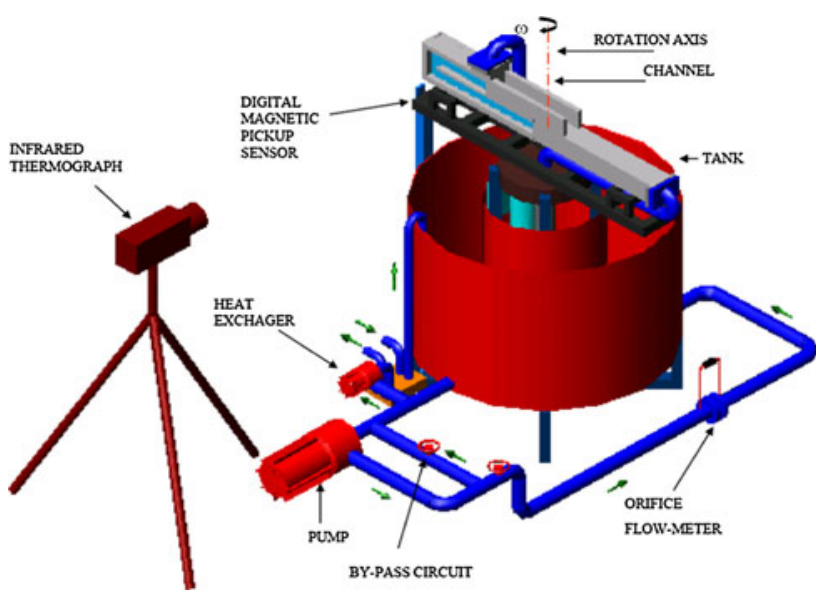

Fig. 24 Apparatus for rotating channel heat transfer measurements. From Gallo et al. (2007) 
Fig. 25 Normalized Nusselt number profiles $(R e=20,000)$ : a leading wall, $\mathbf{b}$ trailing wall. From Gallo et al. (2007)

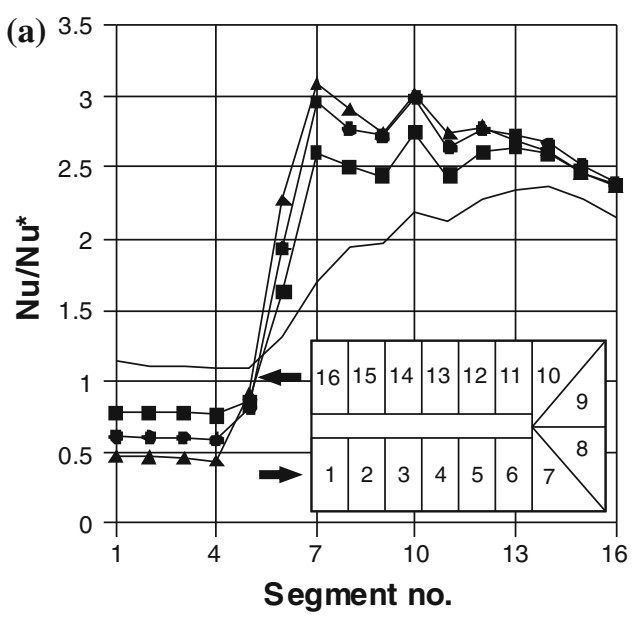

For the leading wall (Fig. 25a), Nu/Nu* values are lower than those relative to the static case and decrease for $R o$ increasing. At the fifth segment, i.e. about one hydraulic diameter before the turn, the Nusselt number starts to increase and reaches a maximum value in segment seven. In fact, in the turn zone, the radial velocity component suddenly decreases and changes sign with a consequent decrease and an inversion of the Coriolis force. This inversion makes the flow to separate by the trailing side and to abruptly reattach toward the leading side, with a strong increase in $N u / N u^{*}$ at reattachment. For all rotation numbers, profiles in the turn region and outlet duct exhibit analogous trends with values of the normalized Nusselt number increasing for rotation number increasing.

At the trailing wall (Fig. 25b) of the inlet duct, the $\mathrm{Nu} / \mathrm{Nu}$ * values are higher than for the static case and slightly increase as $R o$ increases. In the first corner and in the first half of the second corner (segment nos. 7, 8 and 9), the profiles relative to all rotation numbers present the same trend of the curve pertinent to the static case but with higher $\mathrm{Nu} / \mathrm{Nu} u^{*}$ values. For rotation number increasing, in the outlet duct, it is possible to note that the maximum of the convective heat transfer coefficient (located downstream of the leading wall case) increases and tends to move upstream, i.e., toward the tip of the partition wall.

\subsection{Rotating surfaces}

Rotating systems are quite relevant in several applications and the rotating disk is their simplest configuration. As a matter of fact, flywheels, turbine disks to which blades are attached, disk brakes and even modern high-speed CD-ROMs are all examples of practical applications of this model. Often, the fluid resistance due to rotation is irrelevant but there are a number of cases where the disk thermal behavior may be important.
Astarita and Cardone (2008) perform convective heat transfer measurements on a heated rotating disk, with a relatively small centred jet impinging on it. The disk section consists of a printed circuit board glued on a 20-mm-thick polystyrene foam slab contained in a 450-mm-diameter steel cup, which rotates in the range $\omega=100-4,400 \mathrm{rpm}$. Air, passing through a heat exchanger and a rotameter, goes through a nozzle to produce the jet. The heat exchanger ensures a jet bulk temperature practically equal $\left( \pm 0.1^{\circ} \mathrm{C}\right)$ to the temperature of the ambient air the jet mixes with. Several nozzle exit diameters $d$ and nozzle exit to disk distances $z$ are tested. The jet always impinges perpendicularly at the center of the disk.

This topic gives the chance to present authors to report a case where the intrinsic two-dimensionality of IR camera is reduced to a single dimension only, during tests performed with the just described apparatus to detect the spiral vortices (attached to the disk surface) which occur in the transitional regime of a simply rotating disk (with no jet) and cause $h$ azimuthal variations. Since during a full frame acquisition time for the tested conditions, the disk would rotate about two turns thus producing a blurred image, the vortices attached to the surface are detected by the line-scan option of the used Agema 900 IR camera (Astarita et al. 2002b). While the disk is rotating at thermal steady state, the temperature profile along one radius is acquired as a function of time. Then, the temperature map of the disk surface is reconstructed by taking into account its rotation, i.e. by azimuthally displacing the acquired radial profile sequence so as to generate the thermal map attached to the disk surface. Fig. 26 shows such a map, obtained by azimuthally displacing about 30,000 profiles, where the footprint of the vortices is clearly visible. The disk diameter is equal to the side of the externally black square since the temperature range to detect the vortices is very low.

Before treating the disk with the jet, the importance of the adiabatic wall temperature $T_{\text {aw }}$ in high-speed flows is 


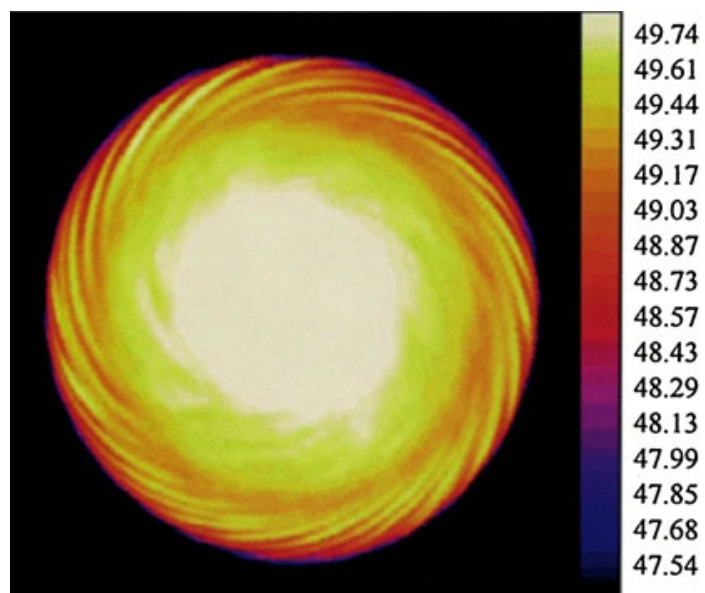

Fig. 26 Reconstruction of spiral vortices on a rotating disk. From Astarita et al. (2002b)

illustrated with thermograms obtained in the normal way (2D) in the same apparatus. The upper half of Fig. 27 (Cardone et al. 1996) shows the thermogram of the disk rotating at 4,390 rpm with a Joule heat flux $Q_{j}=871 \mathrm{~W} / \mathrm{m}^{2}$. A relatively small region ( $\approx 16 \%$ of the disk surface) at the disk center, with a constant wall temperature $T_{\mathrm{w}}$, and where the flow is laminar, is clearly evident. In fact, since the laminar solution of Millsaps and Pohlhausen (1952) predicts that the convective heat transfer coefficient $h$ has to be constant over the surface, from Eq. (10) it follows that, for a constant heat flux boundary condition and constant $T_{\text {aw }}$ distribution, the wall temperature $T_{\mathrm{w}}$ must be constant. Actually, in the low subsonic regime, $T_{\text {aw }}$ coincides with fluid temperature and near disk center speed is low.

Outside the laminar zone, the temperature $T_{\mathrm{w}}$ decreases, first rapidly in the transitional regime and then, more slowly in the turbulent one. Immediately after, the wall temperature trend is reversed as $T_{\mathrm{w}}$ begins to slowly rise

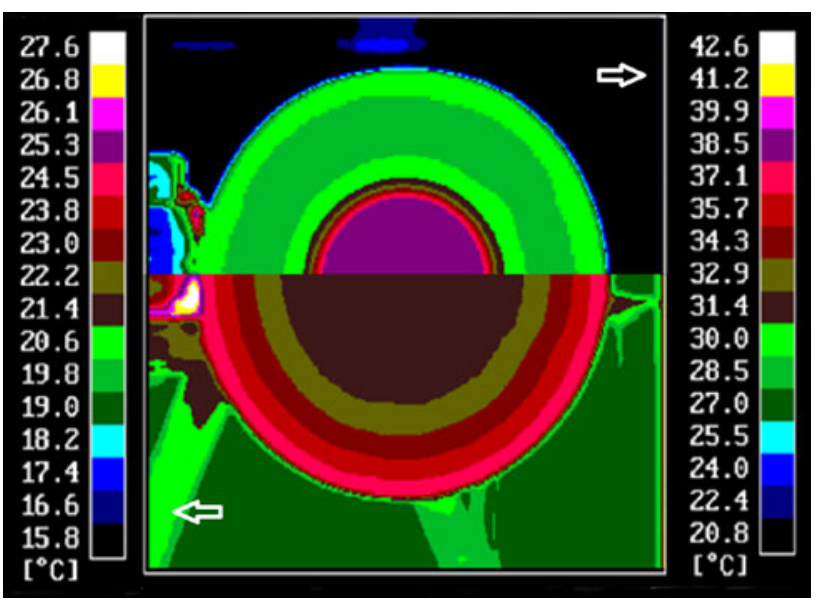

Fig. $27450 \mathrm{~mm}$ disk rotating at 4,390 rpm. Upper half $T_{\mathrm{w}}$ (right scale): $Q_{j}=871 \mathrm{~W} / \mathrm{m}^{2}$. Lower half $T_{\text {aw }}$ (left scale): $Q_{j}=0 \mathrm{~W} / \mathrm{m}^{2}$ while, according to the turbulent correlation law (Cardone et al. 1997b), the convective heat transfer coefficient should increase ( $T_{\mathrm{w}}$ decrease) for increasing local radius.

This behavior is explained by examining the lower half of Fig. 27, which shows the adiabatic wall temperature $T_{\text {aw }}$ map (simply obtained with $Q_{j}=0$ ) at the same disk angular speed. In the map, $T_{\mathrm{aw}}$ is practically constant (and equal to $T_{\mathrm{amb}}$ ) only within the circumference whose radius is about $60 \%$ of the disk radius; afterward $T_{\text {aw }}$ shows a significant increase (about $4^{\circ} \mathrm{C}$ over $T_{\text {amb }}$ near the disk edge). Since for the tested conditions, $T_{\mathrm{w}}-T_{\mathrm{aw}}$ is of the same order of magnitude as $T_{\mathrm{aw}}-T_{\mathrm{amb}}$, Eq. (10) explains the $T_{\mathrm{w}}$ behavior.

Then, the tests of a rotating disk with the centred jet performed by Astarita and Cardone (2008) are described. In order to reduce the number of governing parameters and because of the lack of theoretical analysis, they made the assumption that heat transfer coefficient depends on the ratio between the two momentum flow rates (one due to jet, the other one to disk rotation). By assuming a jet width proportional to the distance from the nozzle and laminar flow over the disk, they put such a parameter in the form:

$\chi=\left(\frac{\mathrm{d} V}{v}\right)\left(\frac{v}{\varpi z^{2}}\right)^{\frac{3}{4}}$

where $v$ is the fluid kinematic viscosity coefficient, $\varpi$ is the disk rotational speed, other quantities being already defined. The convective heat transfer coefficient at the jet stagnation point $h_{o}$ is examined.

By considering only the disk rotation, the flow is always laminar at the disk center and in the case of a jet impinging on it, the $h_{o}$ departure from the single rotation value should be a function of $\chi$ only. Therefore, the dimensionless quantity $h_{o} / k \sqrt{v / \varpi}$, with $k$ fluid thermal conductivity coefficient, is plotted in Fig. 28, where tests, performed by randomly varying disk angular speed, nozzle diameter, jet initial flow rate and nozzle-to-disk distance, are reported as a function of $\chi$.

The two correlation curves, also shown in Fig. 28 with continuous lines are:

$\frac{h_{o}}{k} \sqrt{\frac{v}{\varpi}}=0.33+1.57 \chi ; \quad$ for $\chi<1\left(\rho^{2}=0.988\right)$

$\frac{h_{o}}{k} \sqrt{\frac{v}{\varpi}}=1.81 \chi^{0.597} ; \quad$ for $\chi>1\left(\rho^{2}=0.989\right)$

The two correlations (23) and (24) are, respectively, obtained by using 42 and 122 data points.

\subsection{Micro systems}

Miniaturized systems are being progressively applied in more and more sectors, such as the electronic, 


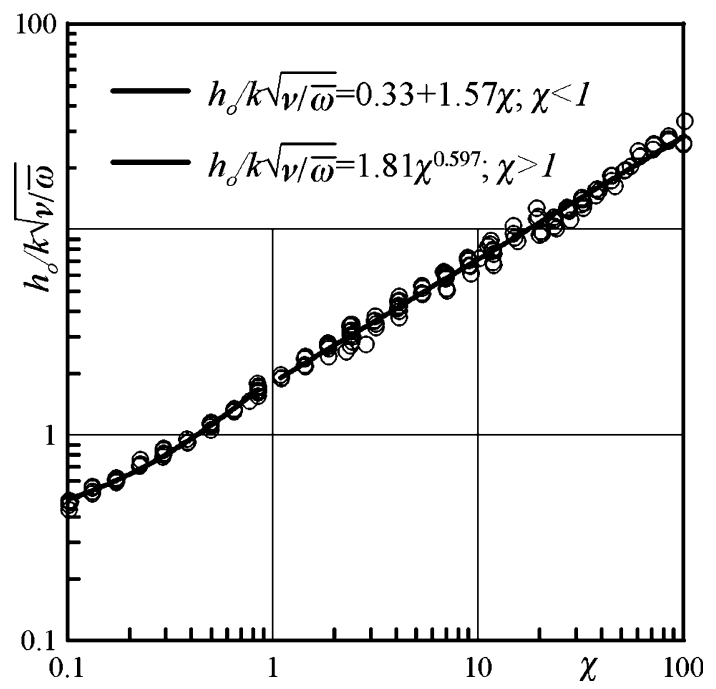

Fig. 28 Convective heat transfer coefficient at the jet flow stagnation point. From Astarita and Cardone (2008)

mechanical, pharmaceutical, and medical industries, as well as in the military sector e.g., for biological and chemical warfare defense. As these areas continue to grow, it becomes more and more important to understand the influence on micro-channel flows of various effects such as entrance length, wall roughness, nano-bubbles on the wall surface, viscous dissipation, electric double layer, etc. Miniaturization, once restricted to space, has become a key word in many advanced technologies as well as traditional industries.

As a general comment, it has to be pointed out that, when using IR thermography for convective heat transfer measurements in micro systems, one has to be very cautious and consider three important aspects. The first one is that the convective heat transfer coefficient $h$ can be very high; e.g., having the Nusselt number for channel laminar flow a constant value, since the tube diameter is small, $h$ increases. The second aspect is that, because of the limited dimensions of the thermal sensor, for non-uniform sensor temperature, tangential conduction may become very relevant. Finally, when working with very small dimensions, another drawback is that, especially for IR cameras working in the LWIR band $(8-12 \mu \mathrm{m})$, the expected spatial resolution easily reaches the system's physical limitations.

As an example of IR thermography application to micro systems, the thermogram of a meniscus (liquid-vapor interface) topping ethanol contained in a vertically oriented capillary tube is reported in Fig. 29 from the work of Buffone and Sefiane (2008). Due to ethanol evaporation, below the meniscus they find by PIV a toroidal vortex (convection cell) where the fluid particles travel along the meniscus from its center toward the capillary walls; from

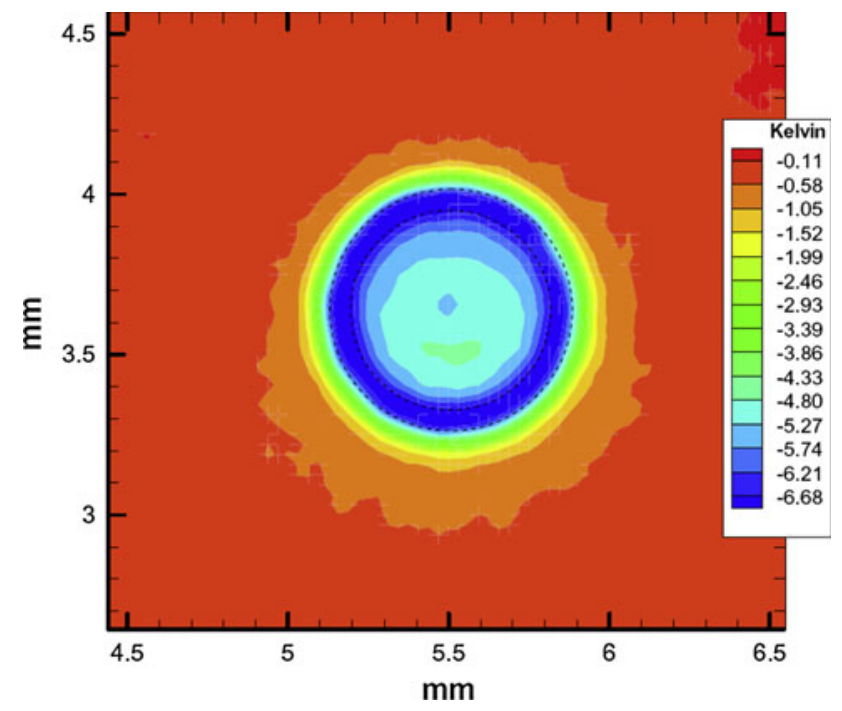

Fig. 29 Thermogram of the meniscus surface of evaporating ethanol inside an unheated tube; indicated temperature is the difference with the ambient one. From Buffone and Sefiane (2008)

there, particles move down along these walls to the bulk phase, returning after to the center and finally, via the capillary axis, back toward the meniscus.

A low resolution scanning camera $(140 \times 140$ pixels $)$ with a microscope (minimum FOV $1.4 \times 1.4 \mathrm{~mm}^{2}$ ) is used to record the thermogram of Fig. 29, where the meniscus surface temperature of evaporating ethanol inside an unheated capillary tube $(600 \mu \mathrm{m}$ ID) is presented; indicated temperature is the difference with the ambient one. The blue circle nearly coincides with the tube wall (100 $\mu \mathrm{m}$ thick), also pinpointed with black broken lines. The thermogram shows a practically constant temperature for large part of the meniscus with strong temperature variations only nearby the tube walls. However, it has to be pointed out that ethanol has a very low contact (wetting) angle and, therefore, the low measured temperatures nearby the walls could be also due to the high viewing angles there (see Sect. 2). Besides the authors do not report any data on ethanol radiative transmissivity even if the convection cell (where temperature gradients are present), whose top they are observing, has a depth of only few hundredths of microns.

\subsection{Two-phase flows}

Flow patterns of the evaporating two-phase flow in heat absorber tubes are of special interest since the tubes are inclined and dryout may occur at low flow velocities because of gravity. Generally, the term dryout indicates a local continuous contact of the gas phase with the pipe surface. In the presence of dryout, large circumferential differences of the heat transfer coefficients result in 
temperature differences, which, in solar collectors, may produce severe thermal stress in the pipe wall and/or damage the covering selective paint. It has to be stressed that two-phase flows often involve unsteady heat transfer so that, for real-time measurements, the choice of the thermal sensor is crucial.

Hetsroni et al. (2003) carry out experiments to study the flow regimes and heat transfer for an air-water flow in $8^{\circ}$ inclined tubes of $25 \mathrm{~mm}$ ID by using high-speed video technique, conductive tomography and infrared thermography. Under the studied conditions, disturbance waves of different forms are observed. The analysis of the heat transfer coefficient behavior, together with flow visualization and conductive tomography shows that, at certain superficial liquid velocity, dryout takes place in the open annular flow regimes, with motionless or slowly moving droplets. Experiments are carried out with a heated-thinfoil sensor $(50 \mu \mathrm{m}$ thick), which has a frequency response of $15-20 \mathrm{~Hz}$ so, being the highest cluster frequency higher, the authors confine their measurements to average values. The difference between wall and flow temperatures never exceeds $20 \mathrm{~K}$.

Figure 30 shows a typical temperature map and the corresponding circumferential heat transfer coefficient distribution $h_{\theta}$ over the periphery of the pipe, in the angle range $0<\varphi<180^{\circ}$ (where $\varphi=0^{\circ}$ is at the tube top). The heat flux is $Q_{j}=8,000 \mathrm{~W} / \mathrm{m}^{2}$, superficial gas velocity is $V_{\mathrm{G}}=36 \mathrm{~m} / \mathrm{s}$ and superficial liquid velocity $V_{\mathrm{L}}=0.016 \mathrm{~m} / \mathrm{s}$. The flow moves from right to left. Color shades of the map (see scale of the upper half of Fig. 30) indicate wall temperature.

The temperature distribution along the pipe perimeter shows a maximum at the top and a minimum at the bottom of the pipe. The circumferential profile of the heat transfer coefficient $h_{\varphi}$, computed according to Eq. (10) where $T_{r}$ is the fluid bulk temperature and $T_{\mathrm{w}}$ the average wall temperature, is shown in the bottom half of Fig. 30. Comparison with other measurements shows that the distribution of heat transfer coefficient corresponds to dryout on the upper part of the pipe.

\subsection{Hypersonic flows}

The shock-wave/boundary-layer interaction (SWBLI) is a widely studied problem of hypersonic flow, its importance stemming from applied problems, such as efficient design of control surfaces, high-speed air inlets or thermal protection systems in re-entry vehicles.

De Luca et al. (1995b) analyze some aspects of SWBLI in a two-dimensional hypersonic wedge flow over a flat plate/ramp configuration. Flow conditions are only geometrically two-dimensional because, due to formation of Goertler type vortices (see Fig. 9), some spanwise heat
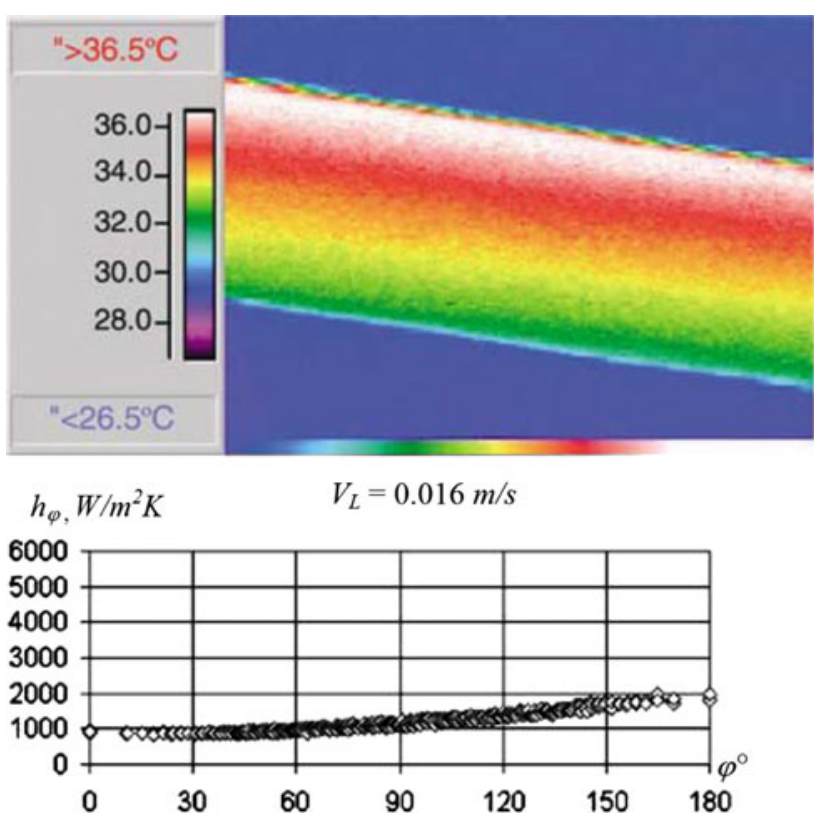

Fig. 30 Thermal patterns on the heated wall (top) and local heat transfer coefficients $h_{\varphi}$ (bottom): $V_{\mathrm{G}}=36 \mathrm{~m} / \mathrm{s}, \quad V_{\mathrm{L}}=0.016 \mathrm{~m} / \mathrm{s}$, $Q_{j}=8,000 \mathrm{~W} / \mathrm{m}^{2}$. From Hetsroni et al. (2003)

flux periodic variations over the ramp at reattaching region are observed. Measurements are performed on a flat plate followed by a ramp in a blowdown wind tunnel at Mach number $M=7.14$ and unit Reynolds number $R e_{\mathrm{u}}=8.6 \times 10^{6} / \mathrm{m}$.

The mean Stanton number $S t$ profiles, measured over a flat plate $(90 \mathrm{~mm} \times 90 \mathrm{~mm})$ followed by a ramp with angles $\alpha$ of $10^{\circ}, 15^{\circ}$, and $20^{\circ}$ are shown in Fig. 31. The increase in the flap angle moves the location of the (laminar) separation upstream. The Stanton number over the ramp attains its highest values for the highest ramp angle of $20^{\circ}$, where the very clear $S t$ peak denotes the presence of a turbulent reattachment. It has to be stressed that Stanton number distributions over the ramp of Fig. 31 refer to spanwise averaged values since, in all of the tested flow conditions, the heat transfer coefficient exhibits a spanwise periodic variation to be ascribed to Goertler type vortices developing in the reattaching flow region.

Cardone (2007) tests SWBLI in a high-enthalpy archeated tunnel which produces $M=6$ flows with a specific total enthalpy up to $2.5 \mathrm{MJ} / \mathrm{kg}$ and a nozzle exit (to the test chamber) diameter of $60 \mathrm{~mm}$. The tunnel operates in a pulsed, quasi-steady mode, with running time up to $200 \mathrm{~ms}$ and the model consists of a flat plate followed by a compression ramp with a spanwise dimension of $100 \mathrm{~mm}$. The hinge line is positioned at $50 \mathrm{~mm}$ from the leading edge, the ramp angle is $15^{\circ}$ and $\mathrm{MACOR}^{\circledR}$ is chosen as model material for its relatively low thermal conductivity, as required to use the thin-film sensor model. A thermal map recorded about $80 \mathrm{~ms}$ after starting of the wind tunnel is 


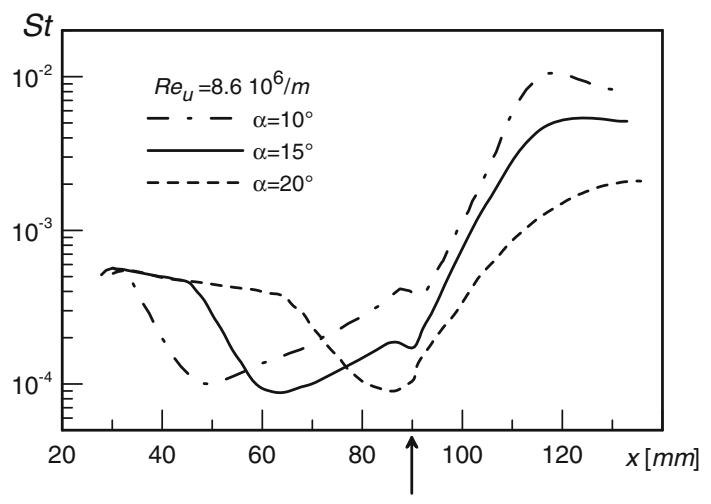

Fig. 31 Streamwise profiles of average Stanton number for a flat plate-ramp configuration and three ramp angles $\alpha$ at $M=7.14$; arrow indicates hinge line position. From de Luca et al. (1995b)

reported in Fig. 32 (flow is from left to right), the temperature distribution being almost two-dimensional only near the model's leading edge. Moving downwind, the continuous decrease in wall temperature shows the boundary layer development. By the hinge line, a region is clearly visible where the temperature attains a minimum due to the presence of a flow separation region. Continuing to move along the symmetry axis, after the hinge line, the temperature reaches a maximum due to flow reattachment on the ramp. Fig. 32 is mainly reported to show how IR thermography allows an easy detection of a non-uniform flow, which, in the present case, is due to the expansion fan starting from the lips of the nozzle exiting in the lowpressure test chamber.

A complex problem regarding hypersonic flow ( $M=5.3$ ) is studied by Gulhan et al. (2008) who report an experimental study on aero-thermo-dynamic effects associated with the injection of a lateral jet into a hypersonic cross flow around a generic missile model, with and without angle of attack. The model consists of a cone, followed by a PEEK ${ }^{\mathrm{TM}}$ cylindrical main body (which includes a single side jet hole normal to the cross stream)

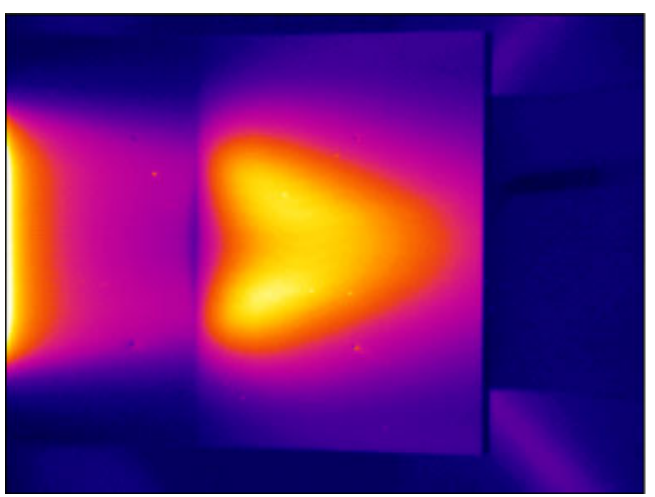

45.36

43.66

41.92

40.15

38.33

36.47

34.59

32.64

30.64

28.60

26.50

24.34

22.11

Fig. 32 Thermogram of a flat plate ramp in hypersonic flow: $M=6$. Flow from left to right. From Cardone (2007) and a flare. Heat transfer measurements, presented in terms of Stanton number distributions, are performed with the thin-film sensor. The flow is also analyzed by oil film visualizations, schlieren images and measuring wall pressure distribution.

The upper part of Fig. 33 shows the Stanton number profiles on the model at circumferential angles $\varphi$ equal to $180^{\circ}$ (jet location) and $150^{\circ}, M=5.3$, angle of attack $\alpha=-10^{\circ}$ (windward injection) and $p_{o j} / p_{\infty}=300$, where $p_{o j}$ and $p_{\infty}$ are the jet total and the free stream pressures, respectively. The lower part of the figure gives the projected oil film image (from the top half of the model) and Stanton number distribution (bottom half) on the model. Downstream of the cone shoulder (placed at $x / D=2.8$ ), the Stanton number drops sharply to a plateau, followed by another drop. As it is evident from the oil film image and the Stanton number map, the separation shock is located at about $x / D=3.6$, downstream of the cone shoulder. The final drop is caused by the separated horseshoe vortex. Upon reattachment of the vortex, the Stanton number profile, for $\varphi=180^{\circ}$, shows a pronounced peak. Downstream of the injection site, the Stanton number rises again, reflecting the fact that the temperature of the jet exceeds that of the free stream. Stanton number profiles for both $\varphi$ angles are similar up to the horseshoe vortex formation. In the area nearby the jet, Stanton number exhibits major differences in the circumferential direction.

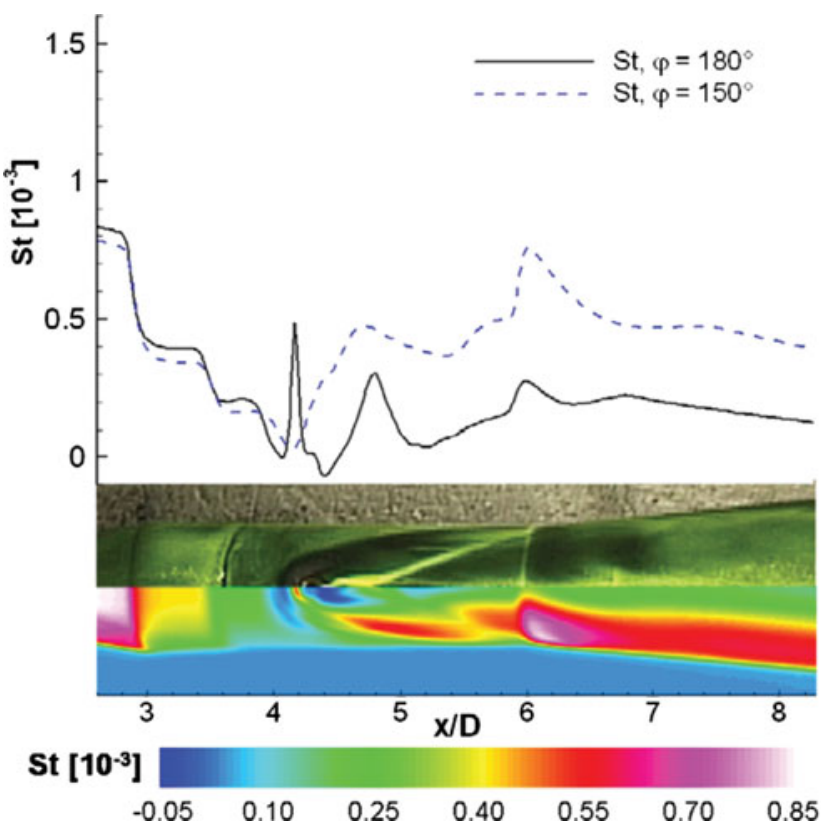

Fig. 33 (Top) Stanton number profiles; (Bottom) Oil film picture (upper) and Stanton number map (lower): $M=5: 3 ; R e=12 \times 10^{6} / \mathrm{m}$; $p_{o j} / p_{\infty}=300 ; \alpha=-10^{\circ}$. From Gulhan et al. (2008) 
At $150^{\circ}$, in the region of the horseshoe vortex wrapped around the jet, the heat transfer is less intense, with Stanton numbers reaching a pronounced minimum there. Farther downstream, outside the area affected by the horseshoe vortex, the Stanton numbers rise markedly due to the impact of the free stream with the flare. The oil film image shows the horseshoe vortex separation line marked by the yellow line that wraps around the fuselage in the outward direction.

\section{Conclusions}

The capability of infrared thermography to be exploited in convective heat transfer measurements is thoroughly analyzed with regard to its various aspects. Since convective heat fluxes measurements must be performed with thermal sensors where temperatures have to be measured with proper transducers, IR camera appears advantageous from several points of view when compared to standard transducers. In fact, IR camera being a fully two-dimensional transducer (up to $1 \mathrm{M}$ pixels), it allows for accurate measurements of surface temperature maps even in the presence of relatively high spatial gradients and, therefore, provides also an easier evaluation of errors due to radiation and tangential conduction. Besides, thermography is nonintrusive (e.g., allowing to get rid of conduction through thermocouple or RTD wires); it has high sensitivity (down to $20 \mathrm{mK}$ ) and low response time (down to $20 \mu \mathrm{s}$ ), which makes it attractive also for rather fast transients. As such, IR thermography can be effectively employed to measure convective heat fluxes with either steady or unsteady techniques.

In this paper, after a short introduction recalling the very main steps of IR thermography history, the basic principles of radiation necessary to perform thermographic measurements are briefly recalled from the user side. Then, an assessment of the main IR camera actual characteristics is presented from the hardware, the performance and the calibration points of view. Afterward, the most commonly used steady and unsteady heat flux sensor models are described. Next, since accurate measurements of convective heat transfer coefficients may be achieved with IR thermography by removing all potential error sources (linked to environment, thermal sensor and IR camera), the problem of thermal restoration and related processing methods is tackled. Finally, a recapitulation table of most of the main contributions appearing in the last two decades is offered and some applications to complex fluid flows, ranging from natural convection to hypersonic regime, are presented and reviewed, also with the aim of pointing out a few relevant aspects connected to IR measurements.
If properly used, IR thermography represents a very powerful optical tool that can be beneficially exploited in complex fluid flows to either measure wall convective heat fluxes or investigate the surface flow field behavior. As such, it gives a wide range of experimental opportunities to fluid-dynamic and heat transfer researchers. Needless to say, IR thermography use is not restricted to thermofluid-dynamics but is actually effectively used also in other fields like electronics, non-destructive material testing, moisture detection, automation, medicine, energy saving, agriculture, predictive maintenance, cultural heritage and environmental applications.

Open Access This article is distributed under the terms of the Creative Commons Attribution Noncommercial License which permits any noncommercial use, distribution, and reproduction in any medium, provided the original author(s) and source are credited.

\section{References}

Abdul Nour RS, Willenborg K, McGrath JJ et al (2000) Measurements of the convection heat transfer coefficient for a planar wall jet: uniform temperature and uniform heat flux boundary conditions. Exp Thermal Fluid Sci 22(3-4):123-131

Abid C, Papini F, Ropke A (1993) Spatiotemporal intermittence of flow in a horizontal cylindrical conduct-determination of the external temperature-field by infrared thermography and study of the behaviour. Journal de Physique III 3(2):255-266

Aliaga DA, Lamb JP, Klein DE (1994) Convection heat-transfer distributions over plates with square ribs from infrared thermography measurements. Int J Heat Mass Transf 37(3):363-374

Astarita T, Cardone G (2000) Thermofluidynamic analysis of the flow in a sharp 180 degrees turn channel. Exp Thermal Fluid Sci 20(3-4): 188-200

Astarita T, Cardone G (2003) Convective heat transfer in a square channel with angled ribs on two opposite walls. Exp Fluids 34(5):625-634

Astarita T, Cardone G (2008) Convective heat transfer on a rotating disk with a centred impinging round jet. Int J Heat Mass Transf 51(7-8):1562-1572

Astarita T, Cardone G, Carlomagno GM et al (2000) A survey on infrared thermography for convective heat transfer measurements. Optics Laser Technol 32(7-8):593-610

Astarita T, Cardone G, Carlomagno GM (2002a) Convective heat transfer in ribbed channels with a 180 degrees turn. Exp Fluids 33(1):90-100

Astarita T, Cardone G, Carlomagno GM (2002b) Spiral vortices detection on a rotating disk. In: Proceedings of 23rd congress international council aeronautical sciences, paper n. ICAS20023.6.4, Toronto

Astarita T, Cardone G, Carlomagno GM (2006) Infrared thermography: an optical method in heat transfer and fluid flow visualisation. Optics Lasers Eng 44(3-4):261-281

Attalla M, Specht E (2009) Heat transfer characteristics from in-line arrays of free impinging jets. Heat Mass Transf 45(5):537-543

Ay HC, Jang JY, Yeh JN (2002) Local heat transfer measurements of plate finned-tube heat exchangers by infrared thermography. Int J Heat Mass Transf 45(20):4069-4078 
Aydin M (1997) Dependence of the natural convection over a vertical flat plate in the presence of the ribs. Int Commun Heat Mass Transf 24(4):521-531

Aymer de la Chevalerie D, Fonteneau A, de Luca L, Cardone G (1997) Gortler-type vortices in hypersonic flows: the ramp problem. Exp Thermal Fluid Sci 15(2):69-81

Bajcar T, Sirok B, Hocevar M et al (2008) Heat transfer influenced by turbulent airflow inside an axially rotating diffuser. Flow Turbul Combust 80(1):3-19

Balageas DL, Boscher DM, Deom AA et al (1991) Measurement of convective heat-transfer coefficients in wind tunnels using passive and stimulated infrared thermography. Recherche Aerospatiale (4):51-72

Baldauf S, Schulz A, Wittig S (2002) High-resolution measurements of local heat transfer coefficients from discrete hole film cooling. J Turbomach Trans ASME 123(4):749-757

Biniwale RB, Kariya N, Yamashiro H et al (2006) Heat transfer and thermographic analysis of catalyst surface during multiphase phenomena under spray-pulsed conditions for dehydrogenation of cyclohexane over Pt catalysts. J Phys Chem B 110(7):3189-3196

Booten CW, Eaton JK (2007) Optically based rapid heat transfer measurements in complex internal flows. J Heat Transf Trans ASME 129(12):1655-1665

Boreman GD (2001) Modulation transfer function in optical and electro-optical systems. SPIE Press, Bellingham, WA (USA)

Bougeard D (2007) Infrared thermography investigation of local heat transfer in a plate fin and two-tube rows assembly. International $\mathrm{J}$ Heat Fluid Flow 28(5):988-1002

Bougeard D, Vermeulen JP, Baudoin B (1994) Spatial resolution enhancement of an IR system by image restoration techniques. EUROTHERM seminar 42, quantitative infrared thermography QIRT 94 Sorrento, Italy, Editions Europennes Thermique et Industrie, pp 3-8

Bougeard D, Vermeulen JP, Baudoin B (1995) Measurement of temperature-field on heat-exchanger fin using infrared thermography - use of a digital restoration technique. Revue Generale de Thermique 34(400-01):A325-A334

Boutarfa R, Harmand S (2005) Local convective heat transfer for laminar and turbulent flow in a rotor-stator system. Exp Fluids 38(2):209-221

Boye H, Staate Y, Schmidt J (2007) Experimental investigation and modelling of heat transfer during convective boiling in a minichannel. Int J Heat Mass Transf 50(1-2):208-215

Buchlin JM (1998) Natural and forced convective heat transfer on slender cylinders. Revue Generale de Thermique 37(8):653660

Buchlin JM (2002) Convective heat transfer in a channel with perforated ribs. Int J Thermal Sci 41(4):332-340

Buffone C, Sefiane K (2004) IR measurements of interfacial temperature during phase change in a confined environment. Exp Thermal Fluid Sci 29(1):65-74

Buffone C, Sefiane K (2008) Controlling evaporative thermocapillary convection using external heating: an experimental investigation. Exp Thermal Fluid Sci 32(6):1287-1300

Burgess NK, Oliveira MM, Ligrani PM (2003) Nusselt number behavior on deep dimpled surfaces within a channel. J Heat Transf 125:11-18

Cardone G (2007) IR heat transfer measurements in hypersonic plasma flows. QIRT J 4(2):233-251

Cardone G, Astarita T, Carlomagno GM (1996) Infrared heat transfer measurements on a rotating disk. Opt Diagn Eng 1(2):1-7

Cardone G, Astarita T, Carlomagno GM (1997a) Heat transfer measurements on a rotating disk. Int J Rotat Mach 3:1-9

Cardone G, Buresti G, Carlomagno GM (1997b) Heat transfer to air from a yawed circular cylinder, chap 10. In: Nakayama Y, Tanida Y (eds) Atlas of visualization III, pp 153-168
Cardone G, Astarita T, Carlomagno GM (1998) Wall heat transfer in static and rotating 180 degrees turn channels by quantitative infrared thermography. Revue Generale de Thermique 37(8):644-652

Carlomagno GM (2006) Colours in a complex fluid flow. Opt Laser Technol 38(4-6):230-242

Carlomagno GM (2007) Heat flux sensors and infrared thermography. J Vis 10(1):11-16

Carlomagno GM, de Luca L (1989) Infrared thermography in heat transfer In: Yang WJ (ed) Handbook of flow visualization, chap 32. Hemisphere, London, pp 531-553

Carlomagno GM, de Luca L (1991) Infrared thermography for flow visualization and heat transfer measurements. In: Proceedings of workshop "Stato dell'arte del rilevamento con camera termiche nella banda 8-15 micron" Firenze

Carlomagno GM, Astarita T, Cardone G (2002) Convective heat transfer and infrared thermography. Ann NY Acad Sci 972:177186

Carlomagno GM, Nese FG, Cardone G et al (2004) Thermo-fluiddynamics of a complex fluid flow. Infrared Phys Technol 46(1-2): $31-39$

Christofferson J, Maize K, Ezzahri Y et al (2008) Microscale and nanoscale thermal characterization techniques. J Electron Pack 130(4):Art. No. 041101

Compton DL (1972) Use of an infrared-imaging camera to obtain convective heating distributions. AIAA J 10:1130-1132

de Luca L, Cardone G (1991) Modulation transfer-function cascade model for a sampled ir imaging-system. Appl Opt 30(13):16591664

de Luca L, Cardone G, Carlomagno GM (1990a) Image restoration in thermo-fluid-dynamic applications of IR digital imagery. Infrared Technol Appl SPIE 1320:448-457

de Luca L, Carlomagno GM, Buresti G (1990b) Boundary-layer diagnostics by means of an infrared scanning radiometer. Exp Fluids 9(3):121-128

de Luca L, Cardone G, Carlomagno GM et al (1992) Flow visualization and heat transfer measurement in a hypersonic wind tunnel. Exp Heat Transf 5(1):65-78

de Luca L, Cardone G, Aymer de la Chevalerie D et al (1993) Goertler instability of a hypersonic boundary-layer. Exp Fluids 16(1):10-16

de Luca L, Guglieri G, Cardone G et al (1995a) Experimentalanalysis of surface flow on a delta-wing by infrared thermography. AIAA J 33(8):1510-1512

de Luca L, Cardone G, Aymer de la Chevalerie D et al (1995b) Viscous interaction phenomena in hypersonic wedge flow. AIAA J 33(12):2293-2298

Dees JE, Bogard DG (2008) Effects of regular and random roughness on the heat transfer and skin friction coefficient on the suction side of a gas turbine vane. J Turbomach Trans ASME 130(4):Art. No. 041012

Dhungel A, Lu YP, Phillips W et al (2009) Film cooling from a row of holes supplemented with antivortex holes. J Turbomach Trans ASME 131(2):Art. No. 021007

di Francescantonio N, Savino R, Abe Y (2008) New alcohol solutions for heat pipes: Marangoni effect and heat transfer enhancement. Int J Heat Mass Transf 51(25-26):6199-6207

Diaz MC, Schmidt J (2007) Experimental investigation of transient boiling heat transfer in microchannels. Int $\mathrm{J}$ Heat Fluid Flow 28(1):95-102

Dizene R, Dorignac E, Charbonnier JM et al (2000) Experimental study of inclined jets cross flow interaction in compressible regime. II. Effect of injection rate on surface heat transfer. Int J Thermal Sci 39(5):571-581

Dorignac E, Vullierme JJ, Noirault P et al (2001) Film cooling: case of double rows of staggered jets. Ann NY Acad Sci 934:337-344 
Dorignac E, Vullierme JJ, Broussely A et al (2005) Experimental heat transfer on the windward surface of a perforated flat plate. Int $\mathrm{J}$ Thermal Sci 44(9):885-893

Ekkad SV, Ou SC, Rivir RB (2004) A transient infrared thermography method for simultaneous film cooling effectiveness and heat transfer coefficient measurements from a single test. J Turbomach Trans ASME 126(4):597-603

Fedrizzi R, Arts T (2004) Determination of the conjugate heat transfer performance of a turbine blade cooling channel. QIRT J 1(1):7188

Feldheim V, Deventer C, Evrard P et al (2005) Local heat transfer coefficient distribution on a plate cooled by an array of confined impinging round jets. QIRT J 2(2):173-190

Fénot M, Vullierme JJ, Dorignac E (2005) Local heat transfer due to several configurations of circular air jets impinging on a flat plate with and without semi-confinement. Int $\mathbf{J}$ Thermal Sci 44(7):665-675

Fénot M, Dorignac E, Vullierme JJ (2008) An experimental study on hot round jets impinging a concave surface. Int $\mathrm{J}$ Heat Fluid Flow 29(4):945-956

Freund S, Pautsch AG, Shedd TA et al (2007) Local heat transfer coefficients in spray cooling systems measured with temperature oscillation IR thermography. Int J Heat Mass Transf 50(9-10): 1953-1962

Gallo M, Astarita T, Carlomagno GM (2007) Heat transfer measurements in a rotating two-pass square channel. QIRT J 4(1):41-62

Gartenberg E, Roberts AA (1991a) Airfoil transition and separation studies using an infrared imaging-system. J Aircraft 28(4):225230

Gartenberg E, Roberts AA (1991b) Mapping flow fields with a heated wire and an infrared imaging-system. J Aircraft 28(4):284-286

Gartenberg E, Roberts AS (1992) Twenty-five years of aerodynamic research with infrared imaging. J Aircraft 29(2):161-171

Gartenberg E, Wright RE (1994) Boundary-layer-transition detection with infrared imaging emphasizing cryogenic applications. AIAA J 32(9): 1875-1882

Gartenberg E, William G, Johnson WG Jr et al (1992) Boundary-layer transition-detection in a cryogenic wind tunnel using infrared imaging. AIAA J 30(2):444-446

Ghisalberti L, Kondjoyan AA (2001) A thermographic method to map the local heat transfer coefficient on the complete surface of a circular cylinder in an airflow. Int J Thermal Sci 40(8):738-752

Goodro M, Park J, Ligrani PM, Fox M, Moon HK (2007a) Effects of mach number and Reynolds number on jet array impingement heat transfer. Int J Heat Mass Transf 50(1):367-380

Goodro M, Park J, Ligrani PM, Fox M, Moon HK (2007b) Separate effects of Mach number and Reynolds number on jet array impingement heat transfer. ASME Transa J Turbomach 129(2): 269-280

Goodro M, Park J, Ligrani PM, Fox M, Moon HK (2008) Effect of hole spacing on jet array impingement heat transfer. Int $\mathrm{J}$ Heat Mass Transf 51(25-26):6243-6253

Goodro M, Park J, Ligrani PM, Fox M, Moon HK (2009) Effect of temperature ratio on jet array impingement heat transfer. ASME Trans J Heat Transf 131(1):1-10 (article 12201)

Gulhan A, Schutte G, Stahl B (2008) Experimental study on aerothermal heating caused by jet-hypersonic crossflow interaction. J Spacecraft Rockets 45(5):891-899

Gunapala SD, Bandara SV, Liu JK et al (2005) 1,024 × 1,024 pixel mid-wavelength and long-wavelength infrared QWIP focal plane arrays for imaging applications. Semicond Sci Technol 20:473480

Gupta A, SriHarsha V, Prabhu SV et al (2008) Local heat transfer distribution in a square channel with 90 degrees continuous, 90 degrees saw tooth profiled and 60 degrees broken ribs. Exp Thermal Fluid Sci 32(4):997-1010
Hedlund CR, Ligrani PM, Moon HK, Glezer B (1999a) Heat transfer and flow phenomena in a swirl chamber simulating turbine blade internal cooling. ASME Trans $\mathrm{J}$ Turbomach 121(4):804-813

Hedlund CR, Ligrani PM, Glezer B, Moon HK (1999b) Heat transfer in a swirl chamber at different temperature ratios and Reynolds numbers. Int J Heat Mass Transf 42:4081-4091

Henckels A, Kreins AF, Maurer F (1993) Experimental investigations of hypersonic shock-boundary layer interaction. Zeitschrift fur Flugwissenschaften und Weltraumforschung 17(2):116-124

Henry RC, Hansman RJ, Breuer KS (1995) Heat-transfer variation on protuberances and surface-roughness elements. J Thermophys Heat Transf 9(1):175-180

Hetsroni G, Hu BG, Yi JH et al (1998a) Heat transfer in intermittent air-water flows-part I horizontal tube. Int J Multi Flow 24(2):165-188

Hetsroni G, Yi JH, Hu BG et al (1998b) Heat transfer in intermittent air-water flows - part II-upward inclined tube. Int J Multi Flow 24(2):189-212

Hetsroni G, Kowalewski TA, Hu B et al (2001) Tracking of coherent thermal structures on a heated wall by means of infrared thermography. Exp Fluids 30(3):286-294

Hetsroni G, Mewes D, Enke C et al (2003) Heat transfer to two-phase flow in inclined tubes. Int J Multi Flow 29(2):173-194

Hozumi K, Yamamoto Y, Fujii K et al (1998) Experimental investigation and numerical analysis of hypersonic compression ramp heat transfer. JSME Int J Ser B Fluids Thermal Eng 41(2):381-389

Inagaki T, Okamoto Y (1999) Measurement of turbulent heat transfer coefficients using infrared thermography near ambient conditions and its quantitative error estimation. JSME Int J Ser B Fluids Thermal Eng 42(2):275-283

Inagaki T, Hatori M, Suzuki T et al (2006) Heat transfer and fluid flow of benard-cell convection in rectangular container with free surface sensed by infrared thermography. J Vis 9(2):145-160

Jain AK (1989) Fundamentals of digital image processing. PrenticeHall International, Inc., Englewood Cliffs

Jalilvand A, Katsuta M, Saitou K et al (2008) The study and development of roll heat pipe performance. Heat Transf Eng 29(12):983

Kim NS, Giovannini A (2007) Experimental study of turbulent round jet flow impinging on a square cylinder laid on a flat plate. Int $\mathrm{J}$ Heat Fluid Flow 28(6):1339

Klein D, Hetsroni G, Mosyak A (2005) Heat transfer characteristics of water and APG surfactant solution in a micro-channel heat sink. Int J Multi Flow 31(4):393-415

Kreins AF, Henckels A, Maurer F (1996) Experimental studies of hypersonic shock induced boundary layer separation. Zeitschrift fur Flugwissenschaften und Weltraumforschung 20(2):80-88

Kutscher CF (1994) Heat-exchange effectiveness and pressure-drop for air-flow through perforated plates with and without crosswind. J Heat Transf Trans ASME 116(2):391-399

Le Sant Y, Marchand M, Millan P et al (2002) An overview of infrared thermography techniques used in large wind tunnels. Aerospace Sci Technol 6(5):355-366

Leger B, Andre P, Grienche G, Schott G (1996) Thermal control of combustion chamber walls. LARA's test bench. Revue Generale de Thermique 35(417):625-663

Li HY, Chen KY (2007) Thermal performance of plate-fin heat sinks under confined impinging jet conditions. Int J Heat Mass Transf 50(9-10):1963-1970

Ligrani PM, Mahmood GI, Harrison JL, Clayton CM, Nelson DL (2001) Flow structure and local Nusselt number variations in a channel with dimples and protrusions on opposite walls. Int $\mathbf{J}$ Heat Mass Transf 44(23):4413-4425 
Liu ZG, Zhao YH, Takei M (2007) Experimental study on axial wall heat conduction for convective heat transfer in stainless steel microtube. Heat Mass Trans 43(6):587-594

Liu ZG, Zhang CW, Zhao XB (2009) Experimental study on influence of microscale effects on liquid flow characteristic in microtubes. Heat Mass Transf 45(3):297-304

Lorenz S, Leiner W, Mukomilow D (1995) Distribution of the heat transfer coefficient in a channel with periodic transverse grooves. Exp Thermal Fluid Sci 11(3):234-242

Lorenz S, Nachtigall C, Leiner W (1996) Permanent three-dimensional patterns in turbulent flows with essentially two-dimensional wall configurations. Int J Heat Mass Transf 39(2):373-382

Loubat R, Reulet P, Estebe B, Millan P (2004) Heat flux characterisation in hot jet and flame/wall interaction by IHCP resolution coupled with infrared measurements. QIRT J 1(2):205-228

Lu Y, Allison D, Ekkad SV (2007) Turbine blade showerhead film cooling: Influence of hole angle and shaping. Int J Heat Fluid Flow 28(5):922-931

Lyons OFP, Murray DB, Torrance AA (2008) Air jet cooling of brake discs. Proc Inst Mech Eng Part C J Mech Eng Sci 222(6):9951004

Mahmood GI, Ligrani PM (2002) Heat transfer in a dimpled channel: combined influences of aspect ratio, temperature ratio, Reynolds number and flow structure. Int J Heat Mass Transf 45:2011-2020

Mai H, Dietz G, Geissler W et al (2008) Dynamic stall control by leading edge vortex generators. J Am Helicop Soc 53(1):26-36

Meinders ER, Hanjalic K (2002) Experimental study of the convective heat transfer from in-line and staggered configurations of two wall-mounted cubes. Int J Heat Mass Transf 45(3):465-482

Meinders ER, van der Meer TH, Hanjalic K et al (1997) Application of infrared thermography to the evaluation of local convective heat transfer on arrays of cubical protrusions. Int J Heat Fluid Flow 18(1):152-159

Meinders ER, Hanjalic K, Martinuzzi RJ (1999a) Experimental study of the local convection heat transfer from a wall-mounted cube in turbulent channel flow. J Heat Transf Trans ASME 121(3):564-573

Meinders ER, van Kempen GMP, van Vliet LJ, van der Meer TH (1999b) Measurement and application of an infrared image restoration filter to improve the accuracy of surface temperature measurements of cubes. Exp Fluids 26:86-96

Menfoukh L, Hanchi S, Oualli $\mathrm{H}$ et al (2008) Experimental investigation of a circular radially deforming cylinder near wake using an infrared technique. Int $\mathrm{J}$ Heat Fluid Flow 29(2):479-494

Meola C, Carlomagno GM (2004) Recent advances in the use of infrared thermography. Meas Sci Technol 15(9):R27-R58

Meola C, de Luca L, Carlomagno GM (1995) Azimuthal instability in an impinging jet-adiabatic wall temperature distribution. Exp Fluids 18(5):303-310

Meola C, de Luca L, Carlomagno GM (1996) Influence of shear layer dynamics on impingement heat transfer. Exp Thermal Fluid Sci 13(1):29-37

Millsaps K, Pohlhausen K (1952) Heat transfer by laminar flow from a rotating plate. J Aeronautical Sci 19:120-126

Mishan Y, Mosyak A, Pogrebnyak E et al (2007) Effect of developing flow and thermal regime on momentum and heat transfer in microscale heat sink. Int J Heat Mass Transf 50(15-16):3100-3114

Montelpare S, Ricci R (2004a) A thermographic method to evaluate the local boundary layer separation phenomena on aerodynamic bodies operating at low Reynolds number. Int $\mathrm{J}$ Thermal Sci 43(3):315-329

Montelpare S, Ricci R (2004b) An experimental method for evaluating the heat transfer coefficient of liquid-cooled short pin fins using infrared thermography. Exp Thermal Fluid Sci 28(8):815-824
Mori M, Novak L, Sekavcnik M (2007) Measurements on rotating blades using IR thermography. Exp Thermal Fluid Sci 32(2):387-396

Mori M, Novak L, Sekavcnik M et al (2008) Application of IR thermography as a measuring method to study heat transfer on rotating surface. Forschung Im Ingenieurwesen-Engineering Research 72(1):1-10

Narayanan V (2007) Oscillatory thermal structures in a reattaching jet flow. J Vis 10(4):389-396

Narayanan V, Patil VA (2007) Oscillatory thermal structures induced by unconfined slot jet impingement. Exp Thermal Fluid Sci 32(2):682-695

Narayanan V, Page RH, Seyed-Yagoob J (2003) Visualization of air flow using infrared thermography. Exp Fluids 34(2):275-284

Narayanan J, Yagoobib S, Page RH (2004a) Transient thermal structure, turbulence, and heat transfer in a reattaching slot jet flow. Int J Heat Mass Transf 47(24):5219-5234

Narayanan J, Yagoobib S, Page RH (2004b) An experimental study of fluid echanics and heat transfer in an impinging slot jet flow. Int J Heat Mass Transf 47(8-9):1827-1845

Novak L, Mori M, Sekavcnik M (2008) Heat transfer study in rotating cascade using IR thermography and CFD analyses. Heat Mass Transf r 44(5):559-567

Patil VA, Narayanan V (2005a) Spatially resolved heat transfer rates in an impinging circular microscale jet. Microscale Thermophys Eng 9(2):183-197

Patil VA, Narayanan V (2005b) Application of heated-thin-foil thermography technique to external convective microscale flows. Meas Sci Technol 16(2):472-476

Patil VA, Narayanan V (2006) Spatially resolved temperature measurement in microchannels. Microfluid Nanofluid 2(4):291300

Peake DJ, Bowker AJ, Lockyear SJ et al (1977) Non intrusive detection of transition region using an infrared camera. AGARDCP-224

Pelle J, Harmand S (2006) Study of heat transfer in a rotor-stator system: impinging jet and spacing influence. J Enhanc Heat 13(4):291-307

Pelle J, Harmand S (2007a) Heat transfer measurements in an opened rotor-stator system air-gap. Exp Thermal Fluid Sci 31(3):165180

Pelle J, Harmand S (2007b) Heat transfer study in a discoidal system: the influence of an impinging jet and rotation. Exp Heat Transf 20(4):337-358

Pelle J, Harmand S (2008) Heat transfer study in a discoidal system: the influence of rotation and space between disks. Int $\mathrm{J}$ Heat Mass Transf 51(13-14):3298-3308

Pelle J, Harmand S (2009) Heat transfer study in a rotor-stator system air-gap with an axial inflow. Appl Therm Eng 29(8-9):15321543

Petre B, Dorignac E, Vullierme JJ (2003) Study of the influence of the number of holes rows on the convective heat transfer in the case of full coverage film cooling. Int $\mathbf{J}$ Heat Mass Transf 46(18):3477-3496

Rahal S, Cerisier P, Azuma H (2007) Benard-Marangoni convection in a small circular container: influence of the Biot and Prandtl numbers on pattern dynamics and free surface deformation. Exp Fluids 43(4):547-554

Reulet P, Donjat D, Divouron E, Radenac E, Millan P (2009) Infrared thermography analysis of the transient aerothermal evolution in a turbofan core compartment model. QIRT J 2(2):225-248

Ricci R, Montelpare S (2005) A quantitative IR thermographic method to study the laminar separation bubble phenomenon. Int J Thermal Sci 44(8):709-719

Ricci R, Montelpare S (2006) An experimental IR thermographic method for the evaluation of the heat transfer coefficient of 
liquid-cooled short pin fins arranged in line. Exp Thermal Fluid Sci 30(4):381-391

Ricci R, Montelpare S, Silvi E (2007) Study of acoustic disturbances effect on laminar separation bubble by IR thermography. Exp Thermal Fluid Sci 31(4):349-359

Riedel H, Sitzmann M (2002) Aspects of flow transition detection when flight testing engine nacelles. J Aircraft 39(6):1019-1027

Roger M (2007) A periodic-transient method for high-resolution heat transfer measurement on two dimensional curved surfaces. J Heat Transf Trans ASME 129(12):1638-1654

Sargent SR, Hedlund CR, Ligrani PM (1998) An infrared thermography imaging system for convective heat transfer measurements in complex flows. Meas Sci Technol 9(12):1974-1981

Schrijer FFJ, Scarano F, van Oudheusden BW (2006) Application of PIV in a Mach 7 double-ramp flow. Exp Fluids 41(2):353-363

Schulein E (2006) Skin-friction and heat flux measurements in shock/ boundary-layer interaction flows. AIAA J 44(8):1732-1741

Seghir-Ouali S, Saury D, Harmand S et al (2006) Convective heat transfer inside a rotating cylinder with an axial air flow. Int $\mathrm{J}$ Thermal Sci 45(12):1166-1178

Sekavcnik M, Mori M, Novak L et al (2008) Heat transfer evaluation method in complex rotating environments employing IR thermography and CFD. Exp Heat Transf 21(2):155-168

Seraudie A, Perraud J, Moens F (2003) Transition measurement and analysis on a swept wing in high lift configuration. Aerospace Sci Technol 7(8):569-576

Shapiro AH (1954) The dynamics and thermodynamics of compressible fluid flow, vol 2. Ronald Press, New York

Simeonides G, Vermeulen JP, Boerrigter HL et al (1993) Quantitative heat-transfer measurements in hypersonic wind tunnels by means of infrared thermography. IEEE Trans Aerospace Electron Syst 29(3):878-893

Sparrow EM, Gregg JI (1956) Laminar free convection from a vertical plate with uniform surface heat flux. Trans ASME 78:435-440

Tarozzi L, Muscio A, Tartarini P (2007) Experimental tests of dropwise cooling on infrared-transparent media. Exp Thermal Fluid Sci 31(8):857-865

Tartarini P, Corticelli MA, Tarozzi L (2009) Dropwise cooling: experimental tests by infrared thermography and numerical simulations. Appl Therm Eng 29(7):1391-1397

Thomann H, Frisk B (1968) Measurement of heat transfer with an infrared camera. Int J Heat Mass Transfer 11:819-826
Veeraragavan A, Cadou CP (2008) Heat Transfer in Mini/Microchannels With Combustion: A Simple Analysis for Application in Nonintrusive IR Diagnostics. J Heat Transf Trans ASME 130(12):Art. No. 124504

Watel B, Harmand S, Desmet B (1995) Convective exchange evaluation on a rotating-disc submitted to an air stream parallel to its surface, by infrared thermography. Revue Generale de Thermique 34(400-01):272-285

Watel B, Harmand S, Desmet B (1999) Influence of flow velocity and fin spacing on the forced convective heat transfer from an annular-finned tube. JSME Int J Ser B Fluids Thermal Eng 42(1):56-64

Watel B, Harmand S, Desmet B (2000a) Experimental study of convective heat transfer from a rotating finned tube in transverse air flow. Exp Fluids 29(1):79-90

Watel B, Harmand S, Desmet B (2000b) Influence of fin spacing and rotational speed on the convective heat exchanges from a rotating finned tube. Int Journal of Heat and Fluid Flow 21(2):221-227

Waye SK, Bogard DG (2007) High-resolution film cooling effectiveness measurements of axial holes embedded in a transverse trench with various trench configurations. J Turbomach Trans ASME 129(2):294-302

Won SY, Mahmood GI, Ligrani PM (2004a) Spatially-resolved heat transfer and flow structure in a rectangular channel with pin fins. Int J Heat Mass Transf 47:1731-1743

Won SY, Burgess NK, Peddicord S, Ligrani PM (2004b) Spatially resolved surface heat transfer for parallel rib turbulators with $45 \mathrm{deg}$ orientations including test surface conduction analysis. J Heat Transf 126:193-201

Yuki K, Okurnura M, Hashizume H et al (2008) Flow visualization and heat transfer characteristics for sphere-packed pipes. J Thermophy Heat Transf 22(4):632-648

Zacksenhouse M, Abramovich G, Hetsroni G (2001) Automatic spatial characterization of low-speed streaks from thermal images. Exp Fluids 31(2):229-239

Zhang C, Song B, Lin YZ et al (2009) Cooling effectiveness of effusion walls with deflection hole angles measured by infrared imaging. Appl Therm Eng 29(5-6):966-972

Zuccher S, Saric WS (2008) Infrared thermography investigations in transitional supersonic boundary layers. Exp Fluids 44(1):145-157 